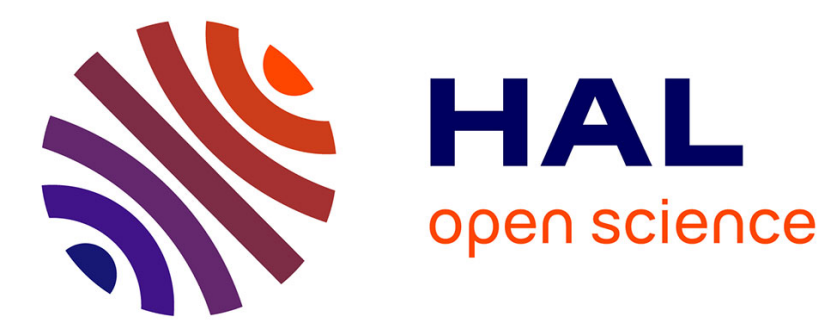

\title{
Un pèlerinage bouddhique au Lanna entre le XVIe et le XVIIe siècle d'après le Khlong Nirat Hariphunchai
}

\author{
François Lagirarde
}

\section{To cite this version:}

François Lagirarde. Un pèlerinage bouddhique au Lanna entre le XVIe et le XVIIe siècle d'après le Khlong Nirat Hariphunchai. Aséanie, Sciences humaines en Asie du Sud-Est, 2004, 14 (1), pp.69 107. 10.3406/asean.2004.1829 . halshs-02545580

\section{HAL Id: halshs-02545580 \\ https://shs.hal.science/halshs-02545580}

Submitted on 17 Apr 2020

HAL is a multi-disciplinary open access archive for the deposit and dissemination of scientific research documents, whether they are published or not. The documents may come from teaching and research institutions in France or abroad, or from public or private research centers.
L'archive ouverte pluridisciplinaire $\mathbf{H A L}$, est destinée au dépôt et à la diffusion de documents scientifiques de niveau recherche, publiés ou non, émanant des établissements d'enseignement et de recherche français ou étrangers, des laboratoires publics ou privés. 


\section{Un pèlerinage bouddhique au Lanna entre le XVle et le XVIle siècle d'après le Khlong Nirat Hariphunchai}

François Lagirarde

\section{Citer ce document / Cite this document :}

Lagirarde François. Un pèlerinage bouddhique au Lanna entre le XVle et le XVIle siècle d'après le Khlong Nirat Hariphunchai. In: Aséanie 14, 2004. pp. 69-107;

doi : https://doi.org/10.3406/asean.2004.1829

https://www.persee.fr/doc/asean_0859-9009_2004_num_14_1_1829

Fichier pdf généré le 05/04/2018 


\begin{abstract}
The Khlong Nirat Hariphunchai $(\mathrm{KNH})$ is one the oldest poems known in Thai literature. Its 180 stanzas tell us about the physical and moral journey of an unknown poet who leaves Chiang Mai and his beloved one to make a pilgrimage to Lamphun (the former Hariphunchai). Eventhough the poet is mainly concerned with his personal sufferings, he gives a detailed account of his religious practices (mainly Buddhist) providing us with a very instructive account of the religious situation in the heart of the Lanna kingdom in its natural and human environment. Most scholars agree on dating this poem from the year $1517 \mathrm{CE}$. But this date seems problematic since it does not match with several facts mentionned by the text itself. For instance $\mathrm{KNH}$ evokes the desertion and ruin of an important number of monasteries - something which, considering what we know about the problems in the Community of monks at this time, points towards a later period. Therefore we think that $\mathrm{KNH}$ should be regarded as a work of fiction weaving together real elements which do not necessarily belong to the same historical period and that a later date (the end of the 16th century ?) should be considered for the composition of the poem.
\end{abstract}

\title{
Résumé
}

Le Khlong Nirat Hariphunchai $(\mathrm{KNH})$ est l'un des plus anciens poèmes de la littérature thaïe. II raconte en 180 strophes le cheminement physique et moral de son auteur, lequel quitte la ville de Chiang Mai et surtout la femme qu'il aime - pour effectuer un pèlerinage de quelques jours à Lamphun (autrefois Hariphunchai). Ce poète inconnu, tout en décrivant la douleur provoquée par la séparation, nous livre une foule de détails sur sa pratique religieuse (avant tout bouddhique) dans son cadre naturel et humain, au cœur du royaume de Lanna. La date de ce pèlerinage, que les spécialistes fixent à 1517 de l'ère chrétienne, pose cependant un problème car elle semble contredite par certaines informations livrées par le document. Ainsi, l'abandon et la ruine de certains monastères tels que décrits dans le texte, semblent renvoyer à une époque postérieure. Ce qui incite à penser que $\mathrm{KNH}$ devrait être considéré comme une œuvre de fiction construite sur des éléments réels mais pas nécessairement tout à fait contemporains - et donc à envisager une date de rédaction plus tardive que celle qui est généralement admise (la fin du XVle siècle ?). 


\title{
Un pèlerinage bouddhique au Lanna entre le $\mathrm{XVI}^{\mathrm{e}}$ et le XVII ${ }^{\mathrm{e}}$ siècle d'après le Khlong Nirat Hariphunchai
}

\author{
François Lagirarde
}

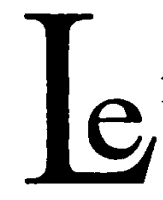

Khlong Nirat Hariphunchai (ou "glon nirāśa haribhuñjăya" en suivant l'orthographe du thaï central, ou, dorénavant, $\mathrm{KNH}$ ) est l'un des plus célèbres poèmes de la littérature thaïe ou "thaïlandaise", pourrait-on dire, puisqu'il s'agit d'une cuvre connue par des versions appartenant à la tradition du Nord (Lanna) et à la tradition du Centre (Siam). KNH est donc représenté par deux familles de textes. La première est formée de manuscrits gravés ou écrits sur feuilles de latanier (bai lan) ou sur cahiers de papier d'écorce de mûrier ( $p h a p s a$ ) dont le texte est en caractères tham ou en caractères nithet et en langue thaïe du Nord. Ces manuscrits sont originaires de Chiang Mai, Chiang Rai et Phayao. La seconde famille est formée de manuscrits écrits en thaï central sur des samut thai dam (livrets "en accordéon" de papier noir). Ils portent un texte siamois peut-être recomposé à Ayutthaya (au XVII siècle) qui ne serait donc finalement qu'une "traduction" ou une adaptation du ou des textes du Lanna'. Aucun manuscrit issu de ces deux traditions n'est cependant antérieur au $\mathrm{XVIII}^{\mathrm{e}}$ siècle alors que le poème lui-même proclame une plus grande antiquité.

1. L'édition critique de $\mathrm{KNH}$ en thaï du Nord a été entreprise à partir de six manuscrits différents (Lamoon 2532,66), le plus ancien provient du Wat Si Khom Kham de Phayao et date de 1758. On connaît quatre manuscrits en thaï central dont trois conservés à la Bibliothèque nationale à Bangkok $\left(n^{\circ} 402,403,405\right)$ à partir desquels ont été publiés quatre éditions imprimées qui ne sont pas considérées comme des éditions critiques au sens technique du terme (Krom Silapakon 2530,4 ). La cinquième édition est celle de Prasert na Nagara qui présente un exemple du texte de Chiang Mai et le texte édité à partir des manuscrits siamois. 


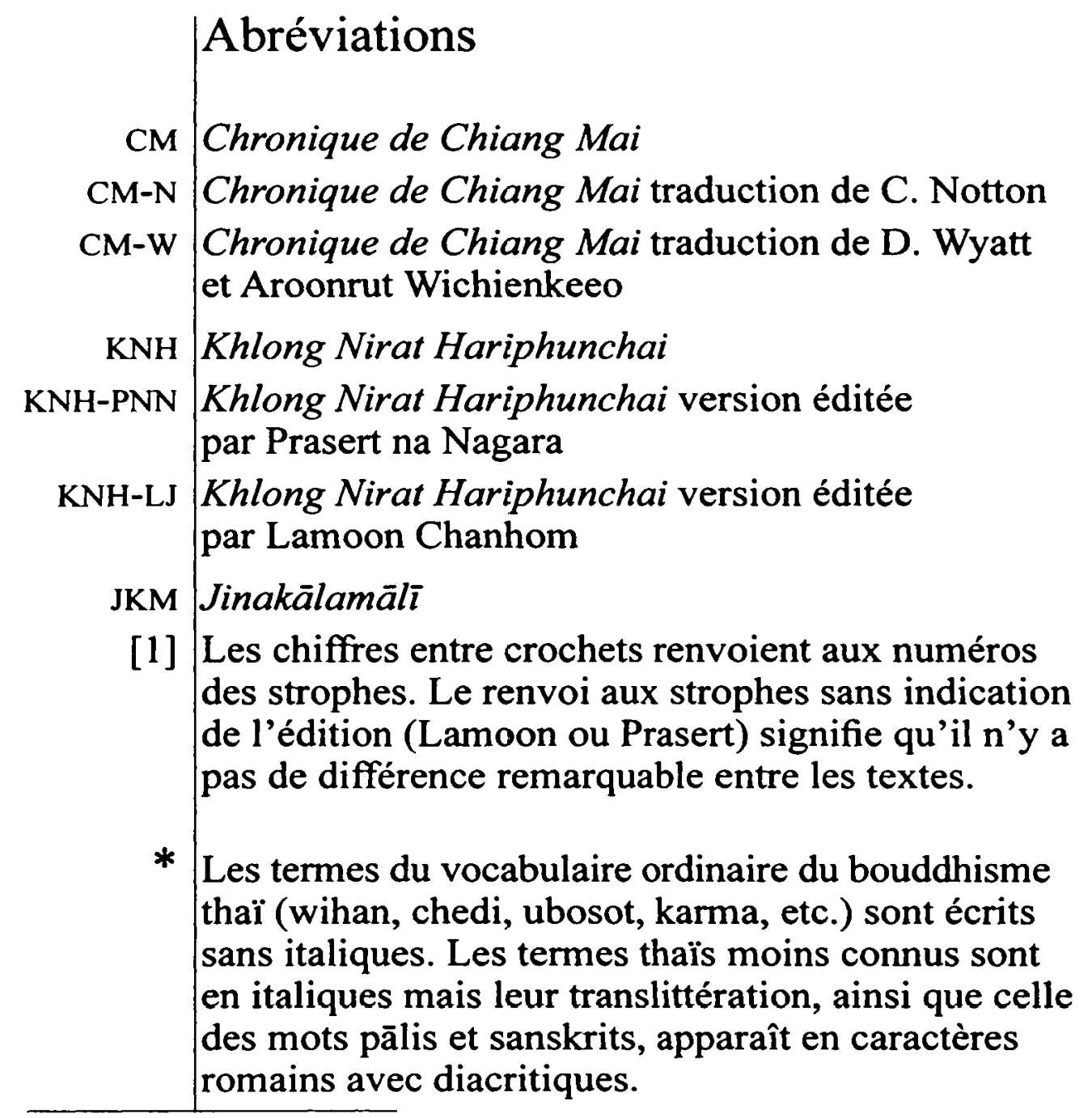


$\mathrm{KNH}$ est un poème mélancolique, écrit à la première personne, qui raconte en 180 strophes assujetties à des règles de versification particulières ( $k$ hlong) le cheminement physique et moral du poète décidé à quitter sa ville de Chiang Mai - mais surtout la femme qu'il aime - pour effectuer un pèlerinage de quelques jours à Hariphunchai (Lamphun). Ce thème du départ et de l'éloignement est, par définition, celui du genre nirat. En réalité, dans le cas qui nous intéresse, le pèlerinage représente l'épreuve qui autorise "la gravité et la solennité d'un départ ${ }^{2}$ ", thème obligé, et peu importe que cette épreuve ne soit finalement qu'assez légère puisque la distance séparant les deux villes n'est que de vingt-six kilomètres en terrain plat. Dans le monde thaii, les pèlerinages ont été et demeurent plutôt des excursions dans les monastères qui permettent de briser la routine pendant quelques jours pour rendre hommage à un sanctuaire, une relique ou un maître importants. En général ces visites pieuses sont joyeuses et nul n'y entend "payer le prix de l'espace" mais ici, répétons-le, ce petit voyage "doit" signifier pour le poète une séparation douloureuse même si son but élevé est de vénérer la Grande Relique du stūpa (cetiya) du monastère principal de Lamphun (le Wat Phra That Hariphunchai). Entre deux dévotions inconciliables dans l'instant, le poète fait donc l'expérience d'un déchirement de l'âme: il y trouve une belle inspiration bien que le choc de ses émotions profanes et de son devoir "sacré" ait quelque chose d'un peu théorique pour le lecteur ordinaire. Mais justement, il n'y a guère de "lecteur ordinaire" de ce texte qui est avant tout un exercice d'écriture extrêmement brillant, une performance stylistique remarquable mais finalement si obscur sur le fond qu'un seul auteur, le professeur Prasert na Nagara, en a proposé une version lisible en thaï moderne qui s'apparente à une véritable traduction commentée. Bref, ce texte énigmatique impose la prudence et nos humbles réflexions sur son contenu dépendent donc totalement du déchiffrement de Prasert.

Au-delà de la définition conventionnelle d'une situation amoureuse banale et au-delà de l'exacerbation littéraire imposée par les prouesses poétiques, on trouve surtout dans $\mathrm{KNH}$ un rapport $a$ priori bien documenté sur les pratiques religieuses - bouddhiques avant tout (mais pas uniquement) - communes dans la région de Chiang Mai à une date qui pourrait peut-être nous ramener à un demi-millénaire dans le passé.

Ces informations sont précieuses pour l'anthropologue des religions pourtant, leur utilisation, de façon historique, demeure délicate car le poème n'est pas véritablement daté. Bien entendu plusieurs dates fixant sa

2. Cette citation et la suivante sont extraites des articles d'Alphonse Dupront réunis dans l'ouvrage Du Sacré, Paris, Gallimard 1987 (p. 373 et 374). 
composition ont été proposées par différents chercheurs. Mais, dans tous les cas, les éléments qui permettent de les avancer font essentiellement partie de la narration elle-même, ce sont des données de la fiction sans aucun lien à un document matériel extérieur - indiscutablement de l'époque - qui pourrait confirmer ou infirmer. Bref, le temps et le lieu proposés sont ceux du récit littéraire plutôt que celui de sa composition ou, mieux encore, celui d'un véritable pèlerinage ayant inspiré la rédaction du poème. Il est donc légitime de se demander si KNH n'est pas partiellement ou totalement une cuvre d'imagination mettant en scène un pèlerinage situé dans un passé idéal, reconstitué pour l'occasion, et permettant de présenter ensemble des événements ou des situations qui ne furent pas nécessairement contemporains les uns des autres. Ce point de vue prudent n'a jamais été adopté par les chercheurs s'intéressant à KNH pour lesquels le temps de la rédaction du KNH original et le temps des "événements" considérés seraient pratiquement le même. Il faut s'empresser de souligner, par ailleurs, qu'il n'y a pas non plus de raisons objectives de douter de l'authenticité de cette poésie, si toutefois ce terme à un sens quelconque en critique littéraire.

C'est aux linguistes que reviendra sans doute un jour le privilège de nous apprendre si oui ou non l'analyse historique, au niveau lexical et phonologique de la langue des deux familles de textes permet de juger de $\mathrm{sa} /$ ses dates proclamées en démontrant les relations précises que ces familles entretiennent. Avec des spécialistes de la versification, ils apprécieront la pureté des vers à l'aune de ce qu'ils savent du développement lexical et tonal des deux langues thaïes en question ${ }^{3}$. En attendant, la lecture et l'analyse des thèmes religieux pourraient peut-être être utiles à double titre. $D$ 'abord pour dresser - certes avec circonspection - un état des lieux des pratiques religieuses des bouddhistes laïcs à une époque "élargie" (sur deux siècles et dans deux capitales). Ensuite, pourquoi pas, pour émettre des hypothèses qui permettraient de resserrer le cadre géo-historique (affiner les dates, résoudre le problème de l'origine du texte) tout en posant la question de savoir pourquoi cette soigneuse description de la vénération bouddhique a été ainsi entreprise si nous pensons bien qu'il y a dans ce texte un au-delà du thème amoureux et des jeux subtils du langage.

3. Car la prosodie thaïe impose des règles strictes en termes de rimes syllabiques et tonales. La question est de savoir comment les rimes tonales ont pu se maintenir au fil des siècles ou malgré le passage d'une langue (le thaï du Nord) à l'autre (le siamois): on lira à ce sujet les remarques fondamentales de W. Gedney $(1989,489-544)$. 


\section{Un texte problématique}

Première difficulté: l'auteur de KNH est inconnu et on ne peut donc préjuger de sa naissance, à la fois en tant que date et origine (quand est-il né, étaitce un Yuan?). Pour les spécialistes de la littérature thaïe ce serait un poète (plutôt de Chiang Mai) fort talentueux et bien informé si l'on en juge par la forme et le fond de son œuvre. Lamoon Janhom $(2538,86)$ a fait remarquer que le style du texte du Lanna est très élaboré et qu'il met en évidence la vaste culture littéraire de son auteur. Non seulement celui-ci est un expert dans la langue thaï $\mathrm{e}^{4}$ mais il semble également familier du khmer, du sanskrit et du pāli. En somme, si cet auteur n'est pas un moine érudit (car son profil linguistique correspond à cette situation), c'est au moins quelqu'un ayant reçu une très bonne éducation, probablement dans le cercle d'une famille royale ou princière. D'un autre côté, on pourrait supposer qu'il s'agit, plus qu'une production royale ou princière directe, d'une noble commande exigeant un travail collectif (ce qui expliquerait la rencontre de l'aspect théorique du drame et de son aspect pratique ou documentaire). Mais ce ne sont ici que pures spéculations et il est plus raisonnable de penser que l'auteur de ces vers restera à jamais un inconnu. Quoi qu'il en soit, il faut sans doute noter en passant que cette science des langues, du khmer et du sanskrit en particulier, nous replace plutôt dans l'orbite culturelle des royaumes ou principautés thaïs situés au Sud du Lanna.

Pour Manas Chitakasem, KNH fut adapté plutôt que véritablement traduit en thaï central au XVII siècle: "converted into Central dialect during King Narai's reign" $(1972,150)$, c'est-à-dire entre1656 et 1688 . Le texte original aurait été composé au siècle précédent à Chiang Mai. C'est le point de vue qui semble dorénavant le plus répandu parmi les chercheurs et c'est sur celui-ci que se fondent nos réflexions et nos questions.

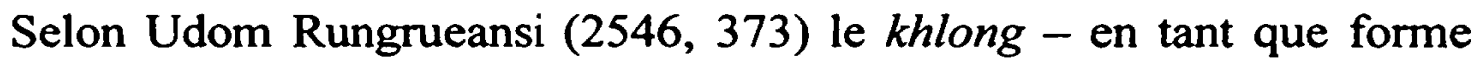
de versification particulière - pourrait même être né au Lanna. Il explique que, bien qu'on ne possède aucune preuve ni aucune indication de l'époque de cette naissance possible, on sait toutefois qu'entre 1367 et 1385 , sous le règne du roi Kuena, le khlong était fort apprécié et que son succès devait perdurer jusqu'à l'époque moderne. Les contraintes imposées par le khlong sont telles qu'il faut faire preuve d'une certaine inventivité lexicale et il est probable que la composition (et la lecture) d'une strophe pouvait apparaître comme un véritable jeu de société basé sur l'échange de jeux de mots et de tournures spirituelles. Cette forme poétique aurait trouvé son apogée entre 1457 et 1657 au Lanna. Lamoon Chanhom $(2538,148)$

4. Il serait sans doute nécessaire de singulariser un peu plus ce "thaî" par rapport à une situation historique du yuan et du siamois mais cela reste à faire. 
rappelle à ce propos que le traité de langue thaïe composé sous le règne du roi Narai (Chindamaṇi) signale des titres de khlong dits "lao" déjà connus à Ayutthaya (vers 1680) (l'adjectif "lao" signifiant ici "ayant pour origine le Nord de l'actuelle Thaillande"). Certains chercheurs, d'accord sur l'idée de l'ancienneté du khlong, font pourtant remarquer que, du point de vue thématique, le genre nirat (poème d'éloignement ou de séparation) appartiendrait plus à la littérature d'Ayutthaya (Hudak 1990, 18). C'était bien l'avis du prince Damrong qui pensait par ailleurs que le terme nirat luimême avait été "prescrit" à l'époque Ratanakosin (cité par Manas 1972, 138). Mais même si le titre complet de KNH était un titre "moderne" on ne peut dire en revanche que le mot nirat était inconnu du poète. On le retrouve utilisé en plusieurs endroits du texte en thaï du Nord $[4,70,174,176,180]$.

Prasert na Nagara date la composition de KNH à $1517^{5}$. Il parvient à cette date précise en combinant deux informations qui dépendent intrinsèquement du récit. La première nous est donnée par le poète qui raconte qu'il est allé rendre hommage au Bouddha d'Émeraude à Chiang Mai avant de prendre la route de Lamphun. Or, selon Prasert, le Bouddha d'Émeraude ne se trouvait à Chiang Mai qu'entre 1468 et $1548^{6}$. Ce premier cadre est lui-même inclus dans un autre, plus vaste: la présence du Phra Puttha Sihing (la statue du Bouddha Sing au monastère du même nom) entre 1407 et $1548^{7}$. La seconde information est une indication de l'année cyclique pendant laquelle le pèlerinage est entrepris. Il s'agit d'une année moeng (cycle dénaire) du bœuf (cycle duodénaire ou des douze animaux) à savoir pi moeng pao. La seule année moeng pao entre 1468 et 1548 tombe alors en 1517 (avril 1517 à mars 1518). On comprendra aisément le problème posé par cette date que rien d'extérieur au texte ne vient corroborer. C'est une possibilité plus qu'une preuve. Les années moeng pao envisageables sont donc 1457, 1517, 1577, 1637, 1697.

Seule la date de 1517 prend effectivement en compte deux données précises du texte. Si le voyage à Lamphun se passe bien à cette date nous nous trouvons alors à l'époque du roi Mueang Kaeo de Chiang Mai qui régna entre 1495 et 1526 . Pour Hans Penth la fin de ce règne laisse percevoir

5. Schweisguth classe KNH dans la littérature siamoise du début du XVII ${ }^{e}$ siècle (1951, 90). Le prince Damrong, cité par Prasert na Nagara, pense à l'année moeng pao correspondant à 1637 (Prasert 2516,12) exactement 120 ans plus tard, car il ne prend pas en compte la présence du Bouddha d'Émeraude à Chiang Mai. Singkha Wannasai préfêre, quant à lui, la date de 1457 .

6. Sur ce point, nulle certitude. Seules les dates du "retour" du Phra Kaeo au Siam et de son installation au wat du même nom, 1778 et 1784 , sont assurées (Penth 1994a, 152).

7. Le professeur $\mathrm{H}$. Woodward Jr. a mis en parallèle, dans un tableau révélateur, la liste des événements associés à ces deux statues combinant les "faits" de mythologie et d'histoire $(1997,504-505)$. 
des signes d'affaiblissement économique du royaume (Penth 1994, 15) sans doute aggravés par l'immense entreprise de reconstruction des murs de brique et de latérite des deux villes de Chiang Mai et de Lamphun entre 1516 et 1517. La Jinakālamālì (Cœdès 1925, 125-126) du moine Rattanapañña donne de très fiables renseignements sur ces années puisque c'est l'époque même où elle fut rédigée. On se rend compte à sa lecture que ces années sont des années de fastes où le roi peut encore se permettre de construire des "murs" d'argent autour du grand stūpa de Lamphun afin de lancer une grande campagne de donation d'or pour ce stūpa.

Des milliers d'ouvriers corvéables ${ }^{8}$ seront employés à ces chantiers mais, curieusement le poète ne signale rien à ce sujet. Singkha Wannasai (cité par Udom Rungrueansi 2542, p. 3291) pense qu'une telle omission n'est pas pensable et que la description de Chiang Mai et Lamphun est antérieure d'un cycle de soixante ans à 1517 . Il propose donc l'année moeng pao 1457. Hélas, $\mathrm{KNH}$ est bien entendu rempli d'omissions que nous trouvons étonnantes a posteriori: s'il est impossible de raisonner sur ces silences, on peut certes les noter.

Un autre élément qui pourrait permettre de dater $\mathrm{KNH}$ nous est donné par la description du Wat Phra Yuen dans les strophes 146 et 150-153. Griswold $(1975,47)$ les a traduites dans la célèbre étude qu'il a consacrée à ce monastère (Wat Phra Yün Reconsidered). Dans la strophe 150 on apprend donc:

I [le poète] cannot see the face of my beloved, I see only the emptiness of an abandoned monastery, where all is withered and dry. The deserted statues remind me of myself.

Ce monastère qui eut une importance considérable est donc abandonné au moment du pèlerinage. Malheureusement on ne sait pas précisément à quelle date, affaibli par les querelles internes de la Communauté, il a été déserté. Griswold suggère que le Wat Phra Yuen fut abandonné au début du XVII ${ }^{e}$ siècle car on a retrouvé des copies des statues du Bouddha debout, des bronzes de facture maladroite mais portant des inscriptions datées de 1537 et 1595 (Griswold 1975, 66). Griswold pense que ces statues furent fabriquées in situ. La dernière (id. fig. 36) fut fondue à la commande du Thera Ananda, sans doute l'un des derniers chefs du Wat Phra Yuen.

On peut en effet souligner le fait que l'abandon du monastère en 1517 semble un peu prématuré et, inutile de le dire, tout à fait invraisemblable en 1457: l'abandon du Wat Phra Yuen fut sans doute causé d'abord par le

8. La JKM évoque en effet la présence de trois millions de travailleurs réquisitionnés pour la muraille de Lamphun (Cœè̀s 1925, 125); même si le chiffre est complètement exagéré les travaux ne durent pas passer inaperçus. 
déclin de la lignée de Sumana Thera puis par l'occupation du Lanna par les Birmans dans un contexte général de marasme économique. Mais à cette époque (1517), comme le montre la JKM, les trois groupes (ou sectes) de bhikkhus sont encore actifs (ils se retrouvent lors des grandes cérémonies organisées par le roi) et la victoire finale de la nouvelle lignée des Sîhāla bhikkhus par voie de réordinations massives n'est pas encore acquise. En 1517 le roi Mueang Kaeo continue de montrer son respect pour les trois traditions sinon de tenter de les réunifier. En cet âge d'or, "Buddhist Pāli literature flourished [...] the three main Buddhist orders (nikai, nikāya) in Lan Na i.g., the two reformed Singhalese orders based in Wat Suan Dòk and Wat $\mathrm{Pa}$ Daeng respectively, and the traditional order based in Wat Phrathat Hariphunchai, were reconciled by Müang Kaeo" (Grabowsky 2004, 19).

Un autre élément troublant apparaît dans la description d'une peinture murale d'un autre monastère abandonné (suññāāām) vraisemblablement situé entre Saraphi et Lamphun [88]. Le poète y découvre des représentations de soldats équipés d'épées et de boucliers et distingue les Thaïs, les Meng (Môns), les Man (Birmans) et les Yiao (Ngiao, Tai Yai ou Shan). Si nous prenons la date de 1517 et retournons plus d'un siècle dans le passé on constate que la Chronique de Chiang Mai note surtout des guerres (et parfois des alliances) avec les "Sudistes" d'Ayutthaya (Siamois), les Yunnanais (Ho), les armées de Nan (les Kao?), les Lao de Luang Phrabang, les Lue (Lü) de Chiang Tung, les Shan et les Lawa (Lua). L'ennemi principal des Yuan, à cette époque, ce sont bien entendu les Siamois et on pourrait penser qu'une peinture murale exécutée au XVI ${ }^{\mathrm{e}}$ siècle fasse bien la distinction entre les deux groupes inconciliables; les Birmans ou Pégouans (commandés par le roi de Pégou) n'entrent vraiment et définitivement en scène qu'en 1558. La mention indistincte des Thaïs est problématique; elle est curieusement répétée un peu après dans le texte qui mentionne la rencontre de moines thaïs dans un pavillon (sala, śālā) ou la découverte d'un sala pour que les moines thaïs viennent y prêcher [94].

KNH est un poème écrit à la première personne: c'est l'œuvre d'un individu qui rencontre ce que nous appelons l'Histoire mais qui ne la prend en compte qu'en fonction du cadre littéraire normatif qu'il s'est imposé, le nirat lui-même. L'expérience du poète - la séparation - qui ne touche que bien superficiellement le lecteur puisqu'elle n'est en rien dramatique - est considérée dans ces vers comme une illustration de la douleur ou de la frustration. Cette douleur (thuk, dukkha), qu'on jugera triviale ou pas (avec notre regard d'Occidental moderne), est un écho de la douleur constitutive qui est la donnée essentielle du discours bouddhique. Vulnérable, le poète construit sa défense comme tous les laïcs le faisaient et continuent de le faire en terre bouddhique: par des actes méritoires (bun, puñña) et prophylactiques qui seront comptabilisés à son bénéfice, éventuellement pour être reversés au compte de sa bien-aimée. 


\section{Le voyage à Hariphunchai au fil du texte 9}

Le pèlerinage a pour but haribhuñj(a) jețtha jina thāna dhātu [3] c'est-àdire le lieu où sont conservées les reliques du Bouddha, le cetiya principal du Wat Phra That Hariphunchai. Le voyage commence dans l'enceinte carrée (?) de la ville de Chiang Mai ici nommée Nabapurī ou Navapurī (prononcer nopbun, "la ville des neuf" ou "la ville neuve ${ }^{10 ") ~[8] . ~ L e ~ p o e ̀ t e, ~}$ qui entre ainsi dans le jeu de la présence et de l'absence, prend le temps de visiter plusieurs monastères de la capitale du Nord avant de la quitter pour de bon.

Le premier monastère visité n'est autre que le célèbre Wat Phra Sing où le poète vient rendre hommage au brah vara jețtha sihinga, le Bouddha Sihing. C'est la statue la plus anciennement vénérée à Chiang Mai: son histoire est malheureusement pleine de vicissitudes et de mystères ${ }^{11}$. On ne sait si l'image actuellement in situ est l'original (d'autres "copies" gardées à Bangkok ou à Nakhon Si Thammarat peuvent y prétendre) car elle a voyagé pour toutes sortes de raisons, bonnes et mauvaises. Elle permet pour certains de donner la date la plus reculée, le terminus a quo, possible du témoignage de $\mathrm{KNH}$ (aux alentours de 1400 pour son installation dans son sanctuaire ou $k u$ ). Pour le poète, cette première pause au Wat Phra Sing lui donne l'occasion d'expliquer le sens qu'il donne à son pèlerinage: exprimer sa dévotion (à un certain nombre d'objets de cultes, tels que des images du Bouddha, des divinités, des maisons d'esprit) faire les dons prescrits et recevoir le "mérite" en retour. Ce mérite, tel est son vœu, doit être transféré à son âme sœur. Toute l'opération est censée faciliter la réunion ultime des amoureux dans un bonheur durable [10-11].

Lorsque le poète quitte le Wat Phra Sing il salue le prasat (prāsāda) du Bouddha Sihing et (ses?) deux lions. La mention du prāsāda semble bien correspondre à une situation historique. La JKM nous apprend en effet que

9. Nous n'avons pas la prétention, dans ce qui suit, de présenter le texte de façon exhaustive mais plutôt d'en extraire des points qui nous semblent remarquables.

10. Nous ne savons pas en réalité à quoi ressemblait le système de défense passive (murs de briques, douves, levées de terre) et donc le plan de la ville de Chiang Mai de l'époque. Lamoon Janhom suppose que le poète commence son "pèlerinage" au milieu de l'après-midi, la veille de la quatrième pleine lune de l'année. Elle pense qu'il passe la nuit au marché de Ton Sai (Ton Rai) à mi-distance entre Chiang Mai et Lamphun pour arriver au Wat Phra That Hariphunchai le jour de la pleine lune (Lamoon 2532, 65).

11. Elle fut même décapitée par des voleurs en 1922. La tête du Bouddha, visible aujourd'hui, a été refaite à cette époque. La chronique Sihinganidāna composée en pāli par le Mahāthera Bodhiranisī célèbre sans doute l'arrivée de la statue à Chiang Mai. Le texte aurait été composé entre 1402 et 1442 (Wyatt $1976,117)$. 


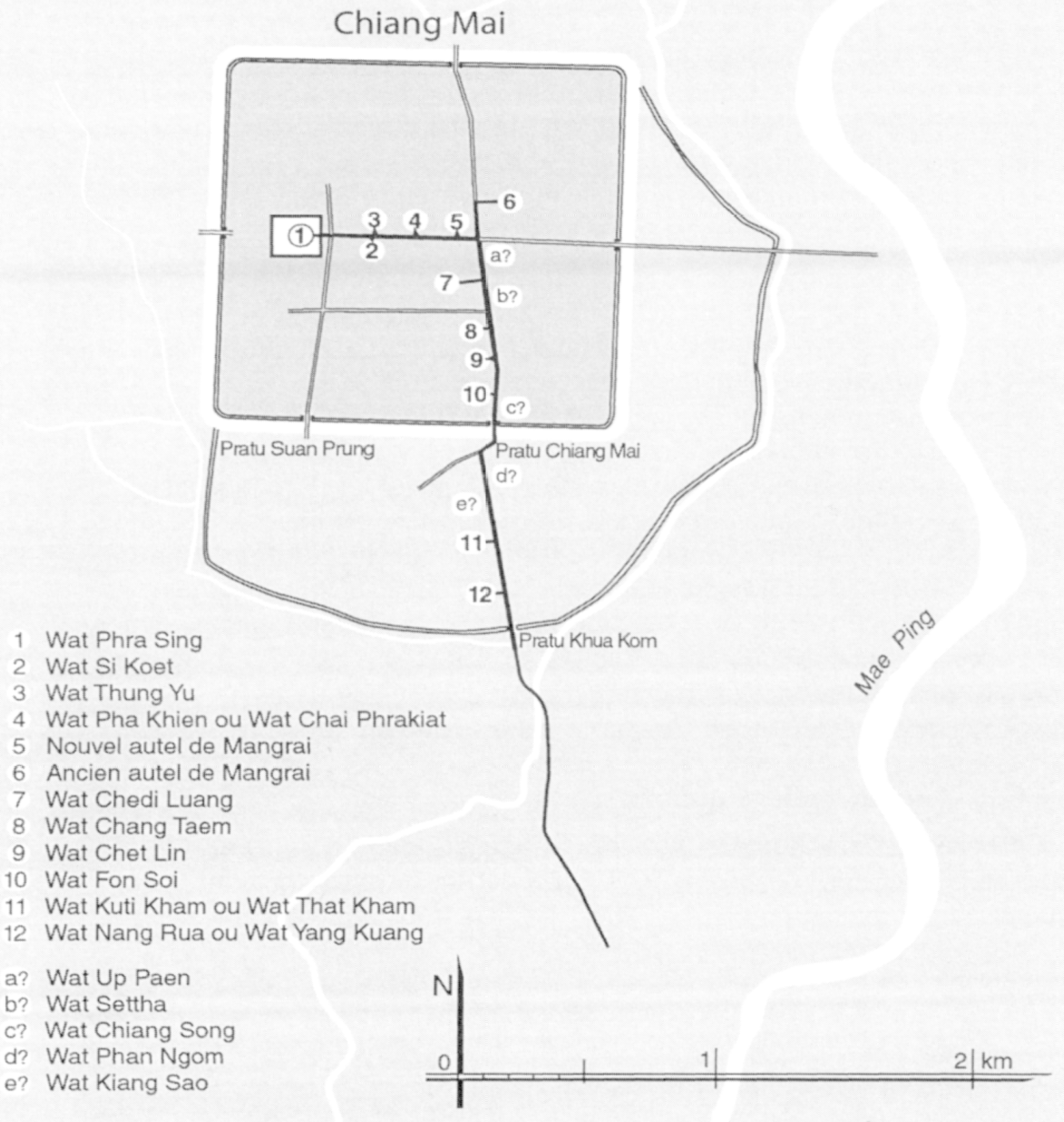

A - Reconstruction de l'itinéraire évoqué dans le Khlong Nirat Hariphunchai: à l'intérieur des remparts de Chiang Mai (dessin P. Pichard) 
1 - L'édifice (prasat ou ku) qui accueillait autrefois la statue du Phra Sihing

Il communique avec le Wihan Lai Kham qui abrite désormais cette statue. (ph. F. Lagirarde, novembre 2004)
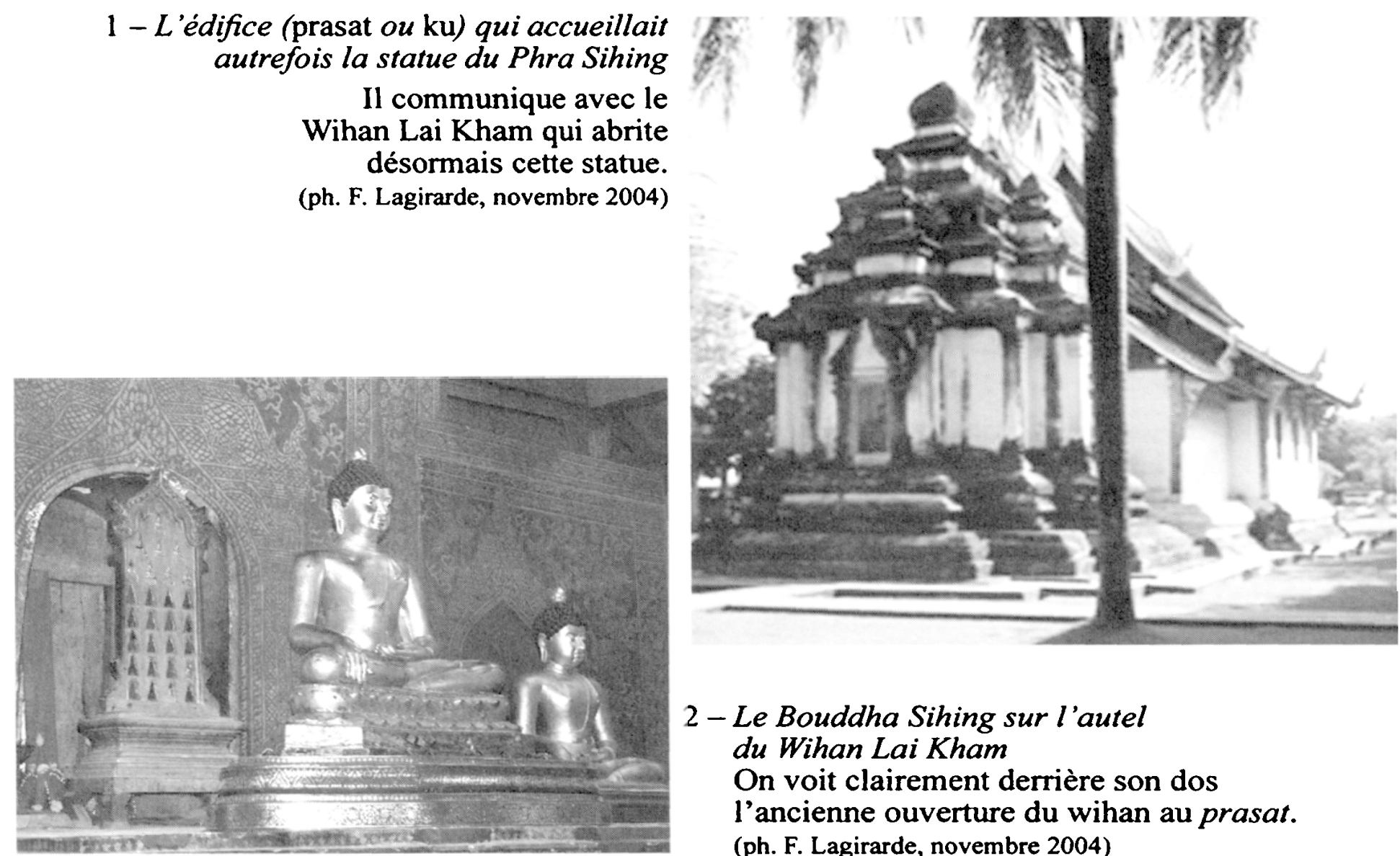

2-Le Bouddha Sihing sur l'autel du Wihan Lai Kham

On voit clairement derrière son dos l'ancienne ouverture du wihan au prasat. (ph. F. Lagirarde, novembre 2004)

cette statue arriva de Kamphaeng Phet sous le règne du roi Kuena (Kü Nā) probablement entre 1355 et 1371 . Ce n'est finalement que sous le règne de son fils, Saen Mueang Ma qu'un édifice fut construit pour la recevoir entre 1385 et 1401 . Cet édifice appelé $k u$ (guhā) est peut-être l'ancêtre de ce prasat de briques qui communique avec l'extrémité Ouest du Wihan Lai Kham, construit, du moins sous la forme que nous lui connaissons, au $\mathrm{XIX}^{\mathrm{e}}$ siècle par le roi Kawila ${ }^{12}$ (fig. 1). Penth fait cependant remarquer que la tradition locale prétend que le roi Mueang Kaeo (r. 1495 et 1526) aurait construit un ou le $k u$ du Bouddha Sihing mais que l'actuel $k u$ attaché au wihan ne date probablement que du XIX ${ }^{e}$ siècle (1994b, 243-244). Toute discussion sur le Bouddha Sihing, il convient ici de le signaler, doit prendre en compte le fait que l'appellation "sihing" définit sans doute plutôt un type, une famille d'images, qu'une statue unique, particulière.

La mention des deux lions est plus problématique. Car si deux animaux fabuleux (des mom) se trouvent bien aujourd'hui des deux côtés de l'entrée du (relativement moderne) Wihan Lai Kham, à une vingtaine de mètres

12. On se fera une idée plus claire de la fonction de ce petit monument, désormais inutilisé, sur les plans et croquis fournis par Chaiyot Itworaphan et Phanuphong Laohasom (2543). Aujourd'hui, le Bouddha Sihing est exposé sur l'autel du Wihan Lai Kham (fig. 2). 
du $k u$ ou du prasat, ce sont des images assez récentes peut-être inspirées d'un modèle chinois ${ }^{13}$. Deux autres mom se trouvent à la porte de l'ubosot qui se trouve tout à côté du wihan (en cours de complète restauration en novembre 2004), deux lions de petite taille en revanche, gardent l'entrée de la bibliothèque (hotrai) et deux autres, plus monumentaux, le grand portail du monastère.

Le poète passe ensuite par trois monastères qu'il désigne sous le nom d'avat (āvasa), le Wat Thung Yu, le Wat Si Koet et le Wat Pha Kian (aujourd'hui Chai Phrakiat) [12]. Il est donc sorti par la grande porte à l'Est du Wat Phra Sing et se retrouve sur l'axe principal (Ouest-Est) de la vieille ville de Chiang Mai. Le Wat Si Koet est à sa droite et les deux autres à sa gauche (au Nord) - du moins à notre époque car on ne possède guère d'informations sur ces trois monastères qui ne sont pas mentionnés dans $\mathrm{JKM}^{14}$. Seul le Wat Si Koet est évoqué dans CM pour des événements relatifs à l'année $1806(\mathrm{CM}-\mathrm{W}, 178)$. Le poète va prendre à droite et arriver au Wat Chedi Luang qu'il appelle Kutaram (kuțārama). Il fait un geste de respect (kraphum) pour obtenir du mérite [13].

Mais (avant d'atteindre véritablement le Wat Chedi Luang) il se trouve à hauteur d'un monastère qui n'existe plus de nos jours (le Wat Up Paen, inconnu) aux environs de l'intersection qui se trouve presque exactement au centre du carré de la ville. Le poète aperçoit également le petit édifice où est vénéré l'esprit du fondateur de Chiang Mai (ho Mangrai), le roi mort en 1317. De cet endroit il fait remarquer qu'émane un "haut" pouvoir surnaturel sung sakti (śăkdi = śăktisiddhi). Il salue (wai) l'autel du roi protecteur (ārakkha rāja) en demandant son aide (bidakșa) et explique alors qu'il accomplit cet acte méritoire dans le but de connaître une demeure céleste et avoir un "[avant-]goût" de l'endroit où vivent les suralai (surālăy) [15]. On suppose qu'un sala se trouvait à ce croisement de routes mais à un emplacement différent de l'actuel san du roi Mangrai qui est de construction très récente (un "sanctuaire" plus ancien existe toujours à quelques centaines de mètres au Nord-Est du croisement dans une allée entre maisons et boutiques).

La prochaine étape se situe dans le Maha Avat (mahā-āvāsa) qui abrite le plus grand chedi de Chiang Mai. Un tremblement de terre devait en détruire la partie supérieure en 1545. C'est bien sûr dans ce monastère, le Wat Chedi Luang, que fut gardée pour un temps la statue de Bouddha la

13. Communication personnelle du professeur Sakchai Saksingha de l'université Silapakon.

14. On date en général le Wat Sri Koet en se référant à $\mathrm{KNH}$, alors qu'on date aussi KNH en se référant à d'autres vestiges archéologiques. La méthode contient le risque d'argumenter (faussement) de façon circulaire. 


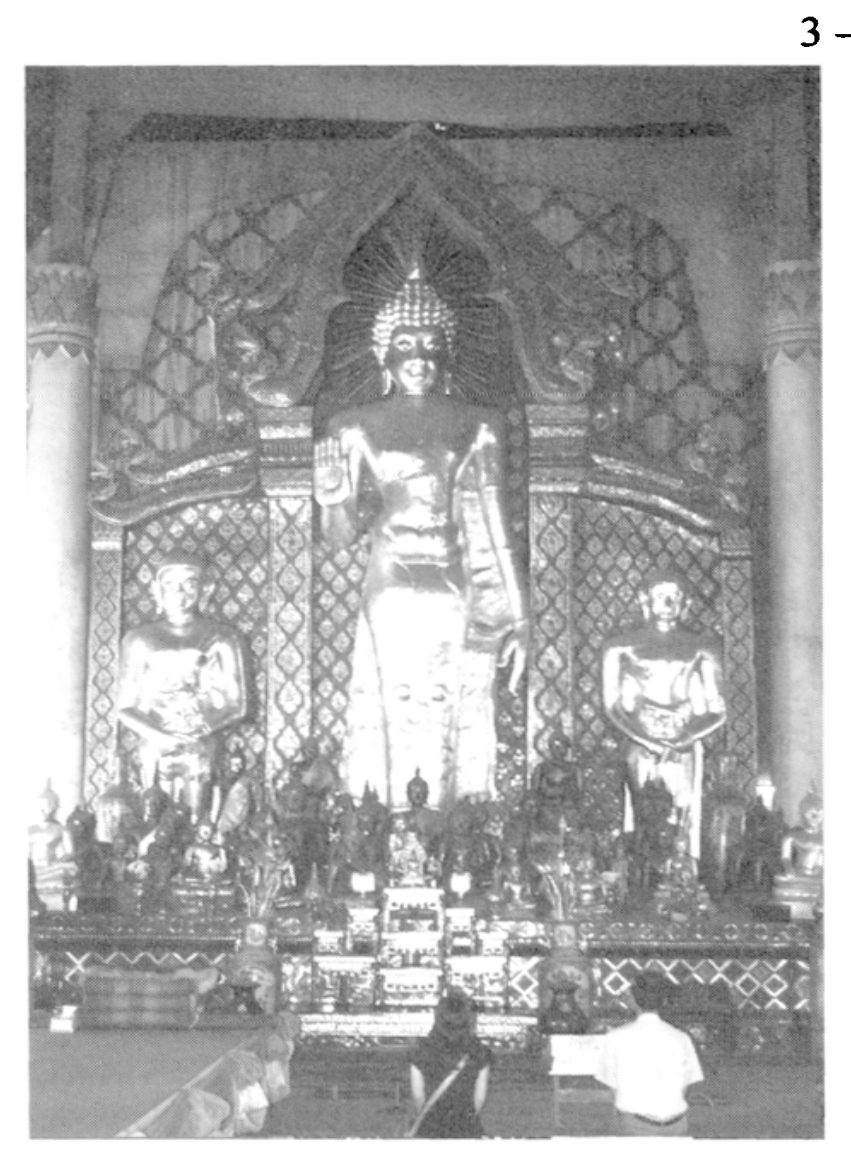

3-Le Phra Attharot

(Bouddha debout) du Wat Phra Sing (ph. F. Lagirarde, novembre 2004)

plus vénérée du monde thaï et lao, ici présentée comme une image du "Jina Ulara" le Grand, le Victorieux (Bouddha) appelé amorakot (amarakata). C'est le Bouddha d'Émeraude qui y aurait demeuré pendant presque un siècle, de 1458 à 1550 , avant de partir avec le roi Setthathirat à Luang Phrabang puis Vientiane [15]. Mais le poète - frustrant ainsi ses lecteurs modernes - ne lui accorde qu'une rapide offrande et un bref salut. On ne saura pas ici dans quel type de sanctuaire se trouvait cette image.

Ensuite, le poète, ayant pénétré dans le grand vihāra central [?] salue le Phra Attharot (brah ățțārasa = une statue du Bouddha debout, haut de 18 coudées ou śàk) [17] cette taille serait celle "du Bouddha de ce monde, le brah bodhi ñāna" (fig. 3). Nous savons par ailleurs que cette image du Bouddha aurait été commandée par la reine Tilokachuda, l'épouse de Saen Mueang Ma en 1411.

Le poète signale également la présence en ces lieux de deux statues d'êtres immortels (amareśa) [18], l'une orientée vers le Nord et l'autre vers le Sud du monastère. Elles sont appelées yakkha rāja, rois des démons porteurs de sabre (khaggā). Ce sont des yak comme il y en a tant dans les monastères de Thaillande. Le poète les interpelle par ces mots: " $\bar{a} \bar{a}$ deva rāja răk eh!" (eh eh, protégez-moi!). Aujourd'hui encore des statues de ces 
redoutables gardiens sont visibles autour du bâtiment dans lequel se tient la colonne d'Indra (ou lak mueang) dans le Wat Chedi Luang. Mais, dans $\mathrm{KNH}$, il doit être question d'autres gardiens puisque ce bâtiment et ces yak furent installés à la demande du roi Kawila, au début du XIX ${ }^{\mathrm{e}}$ siècle $^{15}$.

Le poète rend ensuite hommage (nop ou krap) [19] à une statue du brah sețțī (le Bouddha?) dans un monastère nommé Setțhā. Il se sent déjà très triste de s'être ainsi éloigné de l'être aimé (bien que cet éloignement puisse encore être compté en mètres et en minutes). Il trouve encore sur sa route le Wat Chang Taem puis le Wat Chet Lin (aujourd'hui en ruines) où il remarque un beau sala [20].

L'aram (ārama) suivant est le Wat Fon Soi où il admire une image du Bouddha qu'il appelle jit (jetțha = le suprême). Le poète n'hésite pas à faire un curieux parallèle: cette statue particulière est le rappel du Bouddha historique tout comme chaque jeune fille rencontrée est pour lui l'évocation de sa fiancée, la plus belle de toutes [21]. À présent, il ne reste plus qu'un stūpa en partie écroulé sur l'emplacement de ce monastère (Aroonrut 2539b, 73 et fig. 46) tandis que de nouveaux bâtiments ont été construits. Mais à cette époque, le poète signale l'élégance du décor architectural de ses bâtiments: cho fa (jàn $\mathrm{B} \bar{a}_{2}$ ) ou cornes faîtières à tête de nagas, garudas et autres divinités à large bouche (otthha). Il fait le vœu qu'au sein de ce prasat son acte méritoire (bun, puñña) initié par compassion (karunāa) lui permettra de connaître la prospérité [23]. Après avoir quitté ce monastère il dépasse un certain Wat Chiang Song (non identifié, probablement dans les environs de la porte Sud de Chiang Mai). Sa tristesse devient plus profonde car, dit-il, ses yeux ne peuvent plus apercevoir la bien-aimée.

Le poète quitte alors le périmètre carré de la première enceinte de la ville par une "porte d'or" (dvāra dàn), c'est-à-dire l'actuelle Pratu Chiang Mai, située au premier tiers Est du mur Sud (fig. 4). Il salue l'esprit qui en est le gardien et qu'il appelle mahesak sāt (mahesakkha sādhaya = un devata (?) possédant de grands pouvoirs) le priant d'alléger sa peine (phān). Puis il lui demande (vān) de le soulager de son anxiété (tam ngon, tăṃ , nan) et de l'aider à retrouver celle qu'il aime [25-26]. Un autel (san chaopho pratu chiang mai) existe d'ailleurs à cet endroit de la muraille. Répétant qu'il a quitté la ville par sa grande porte (purī dvāra, srī mahā dvāra), toujours accablé de douleur [27], il atteint un nouveau monastère (Wat Phan Ngom, non identifié) près d'un cimetière (susān) sans doute un de ces terrains où se faisaient des crémations éventuellement des enterrements. Il salue (wai) le Vainqueur de Mara (Jina Māra) [28] et déclare qu'il ne désire recevoir aucune richesse dans une prochaine vie (hurăn / huram) mais plutôt y retrouver encore sa bien-aimée.

15. On trouvera plus de détails, ainsi que des photographies, dans ma présentation de la réimpression de la chronique de Suvanna Khamdaeng traduite par Camille Notton (Lagirarde 2002, 83 et 89). 


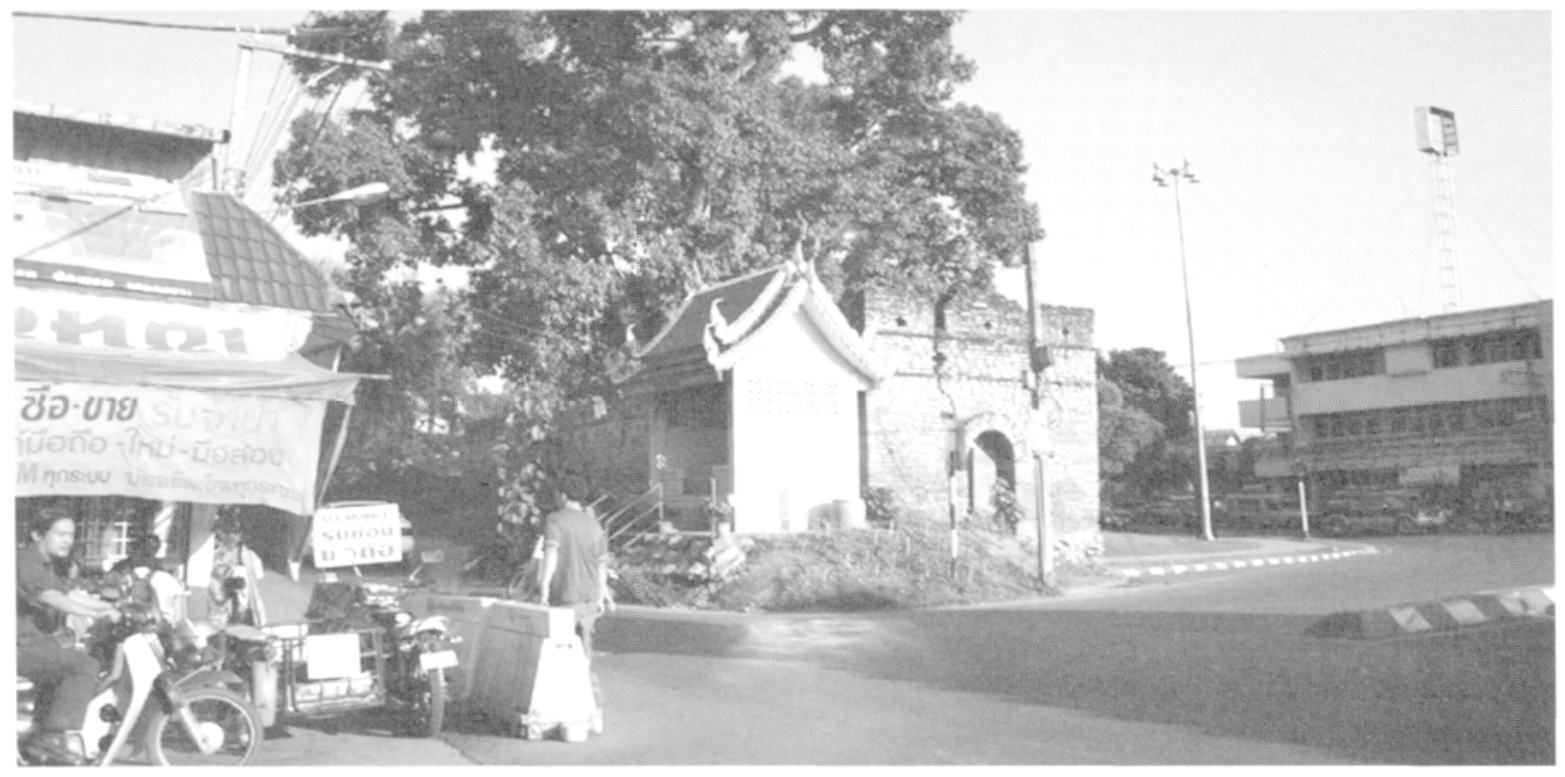

4 - La Porte de Chiang Mai

(Pratu Chiang Mai)

et le petit sanctuaire

de son gardien

(ph. F. Lagirarde,

novembre 2004)

5 - Le chedi abandonné du Wat Nang Rua (Wat Yang Kuang)

(ph. F. Lagirarde, novembre 2004)

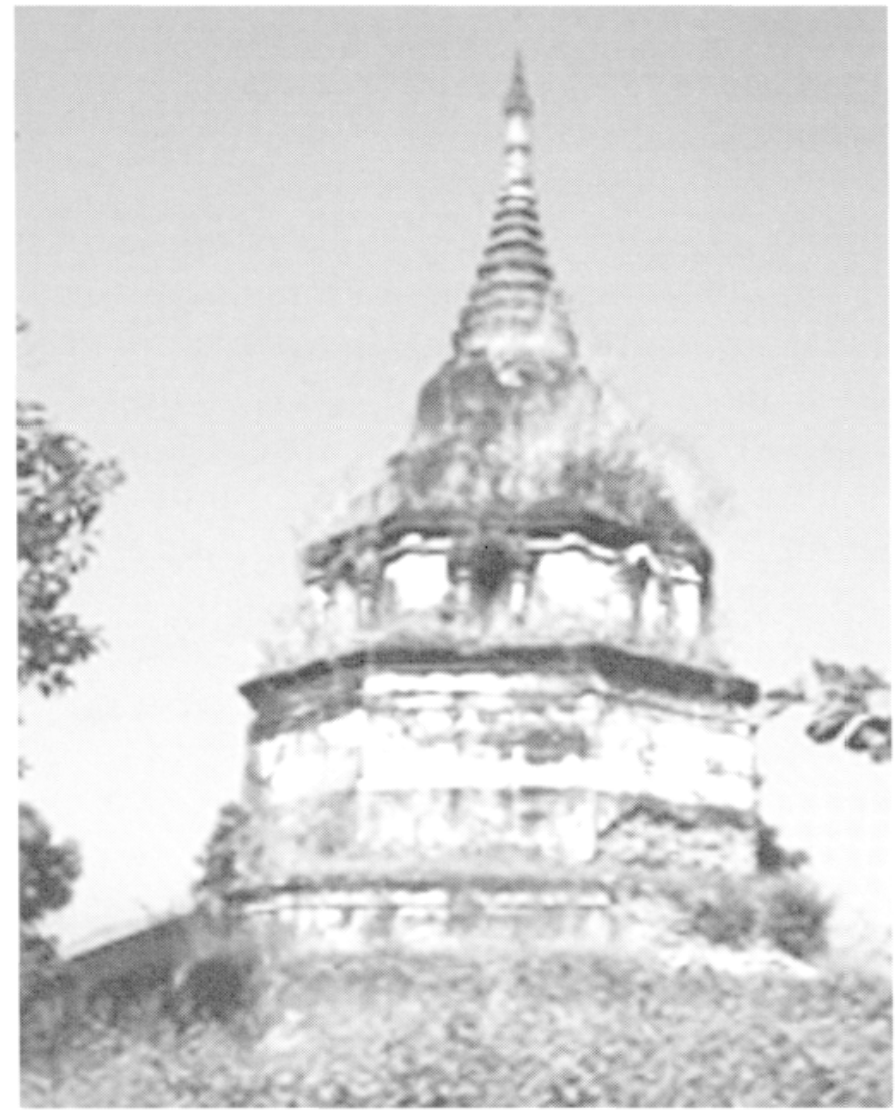

Continuant ainsi vers le Sud par l'actuelle rue Suriyawong, le poète parvient au Wat Kiang Sao (non identifié), puis à ce qu'il présente comme un beau monastère (āvāsa $\mathbf{k e v}_{2}$ ) le Wat Kuti Kham (connu aujourd'hui sous le nom de Wat That Kham dans la rue Suriyawong) [30]. Enfin il évoque un autre splendide monastère, le Wat Nang Rua (fig. 5) qui, selon Lamoon Janhom $(2532,64)$, n'est autre que le site ruiné du Wat Yang Kuang, toujours 


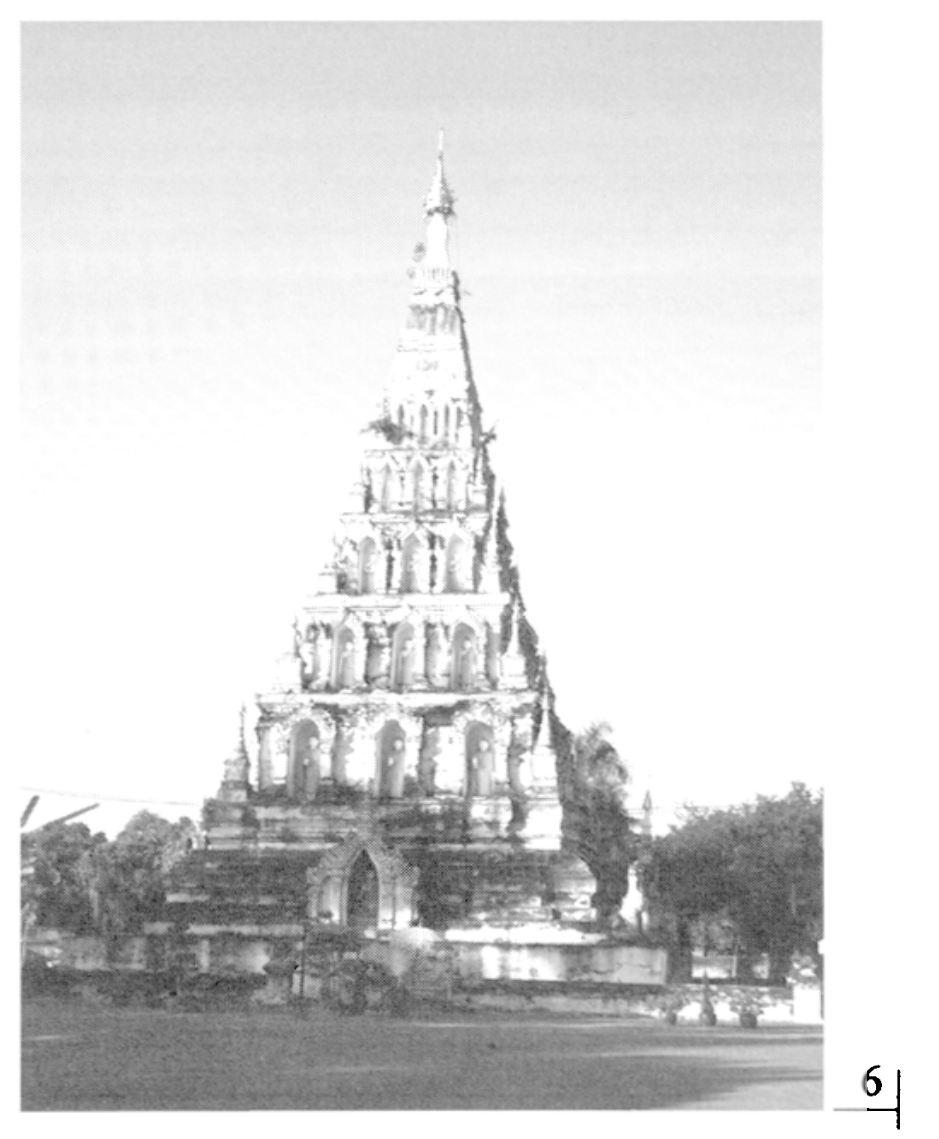

6 - Le chedi du Wat Ku Kham Luang (aujourd'hui Wat Chedi Liam) à l'entrée nord de Wiang Kum Kam

7 - Six statues du Bouddha (sur neuf) sur l'un des quatre côtés du chedi du Wat Ku Kham Luang (photos F. Lagirarde, novembre 2004)

sur la rue Suriyawong ${ }^{16}$. Le poète fait alors remarquer que ce monde est encore bien loin d'une atteinte du nibbāna révélant ainsi sa croyance que ce but ultime n'est pas uniquement celui des religieux engagés définitivement dans la pratique [31]. Il passe une dernière porte d'or [32] sans doute équivalente à la moderne Khua Kom et se trouve alors véritablement en dehors de la ville (buri, purī). Tandis que les rayons du soleil se font moins forts, il prie l'astre diurne de l'aider à éteindre sa souffrance (dap thuk, tăp dukkha) [33]. La mention de cette seconde porte d'or qui, si nous raisonnons avec la datation de Prasert, serait donc déjà bâtie au début du $\mathrm{XVI}^{\mathrm{e}}$ siècle est troublante. Ce ne peut qu'être une porte à travers la seconde enceinte "fortifiée" de Chiang Mai (kamphaeng din, une levée de terre qui fait une courbe allant du coin Sud-Est au coin Nord-Est de la ville carrée) sur laquelle les historiens ont déjà débattu sans parvenir à une conclusion définitive quant à la date de sa construction. Saratsawadi Ongsakun a cependant avancé l'idée que, selon sa propre interprétation des chroniques locales (Tamnan phuen mueang lanna chiang mai), cette deuxième enceinte aurait probablement été édifiée pendant le règne de Thao Maeku entre 1551 et $1564(2529,114)^{17}$.

16. Voir description et photographie de ce monastère in Aroonrut 2539b, 25 et 83-84.

17. Pour appuyer cette hypothèse $\mathrm{CM}$ raconte comment, en 1546 , les armées d'Ayutthaya attaquèrent Chiang Mai d'abord par l'Ouest, le Nord, l'Est puis par le Sud, (CM-N p. 156) attaquant directement la porte Saeng Phung de l'enceinte carrée (aujourd'hui Suan Prung, CM-W p. 112). Ce qui suggère que la seconde enceinte n'existait pas. 


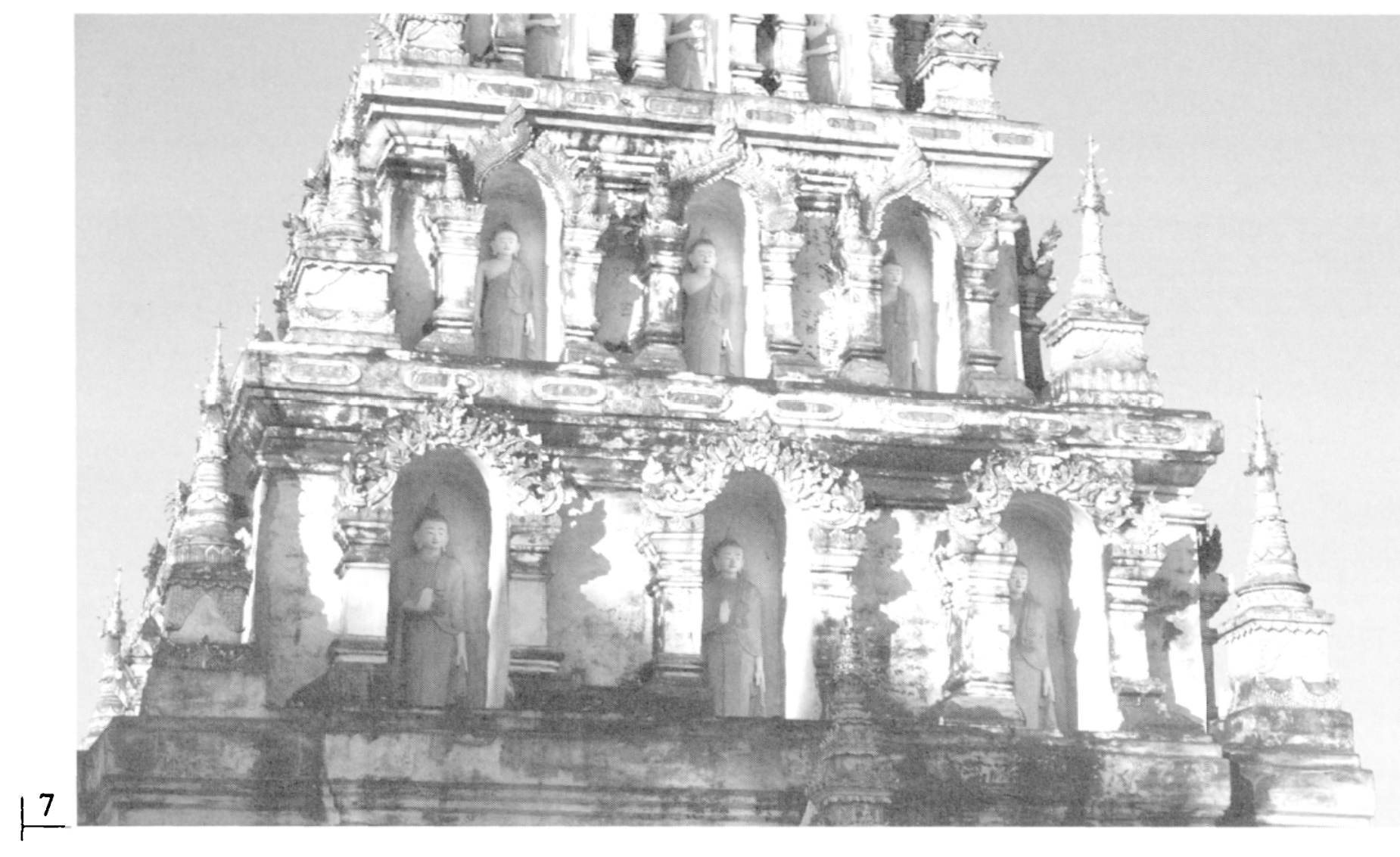

Le voyage continue dans une charrette à bœufs et le poète va atteindre Wiang Kum Kam (fig. 6), hélas sans sa belle [44]! Il arrive aux environs d'un nouveau monastère (āram) installé dans un environnement remarquablement frais (ce qui signifie en général ombrage et verdure). C'est le Wat $\mathrm{Ku}$ Kham Luang construit à la demande du roi Mangrai lors de la fondation de Wiang Kum Kam en 1303 (JKM) ou plus raisonnablement en 1286 avant la fondation de Chiang Mai (Penth 1994b, 86 et 208-209). Il est connu à présent sous le nom de Wat Chedi Liam. Le poète admire les soixante statues du Bouddha (fig. 7) placées dans des niches installées sur les quatre côtés du cetiya (ce type de cetiya "angulaire" [liam] est souvent appelé $K \bar{u}_{1}$ ou $G \bar{u}_{1}$ au Lanna). Il les appelle siamphu (du sanskrit svayambhū = ce qui existe par soi) [45] et observe ensuite une statue en stuc du Bouddha (Jina) assis en samādhi. Il rend hommage (wai) demandant encore la réunification de son couple en s'adressant d'abord à la statue, ensuite à l'esprit protecteur de la reine de Mangrai (dont les cendres sont supposées être enfouies dans le cetiya) [47]. Il se demande pourquoi il connaît un tel malheur. Quelle action (kamma) commise dans une vie antérieure aurait provoqué cette situation? Aurait-il retiré des œufs d'un nid ou détruit un reliquaire (les deux interprétations sont possibles à partir du mot "sārirañ") [48].

Alors qu'il passe sur le site de l'ancienne cité de Kum Kam, le poète, dans une phrase sybilline, évoque les flots (annop, aṇnava) barrés et contenus (khankhop) par un Bouddha couché (phra khwang). Outre que le terme "phra (brah)" n'apparaisse que dans certains manuscrits l'interprétation est fort difficile. On y voit d'abord l'expression d'une idée générale selon laquelle le Bouddha possède le pouvoir d'intervenir sur l'état des choses 

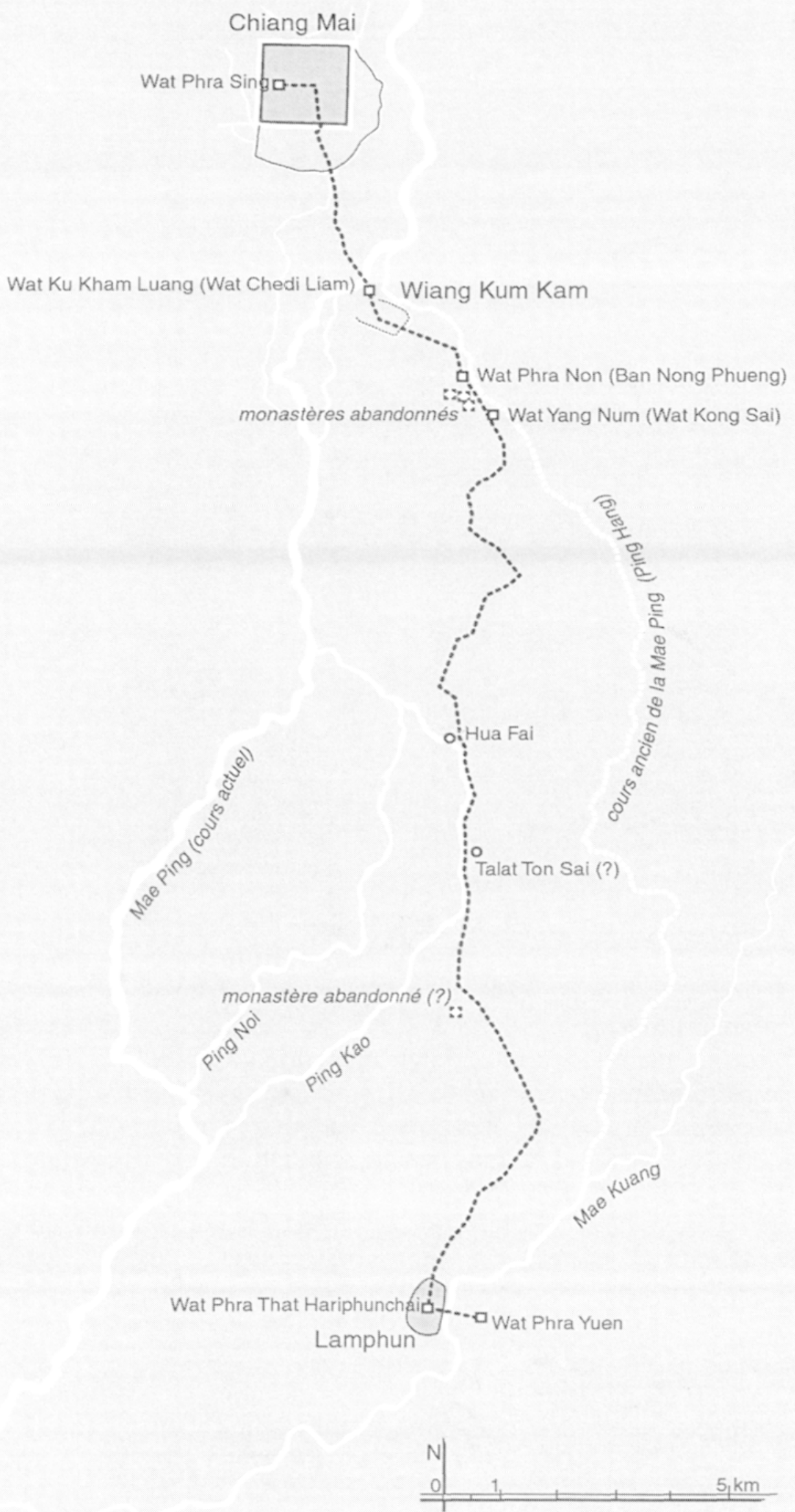

B - Reconstruction de l'itinéraire évoqué dans le Khlong Nirat Hariphunchai: de Chiang Mai à Lamphun (dessin P. Pichard) 


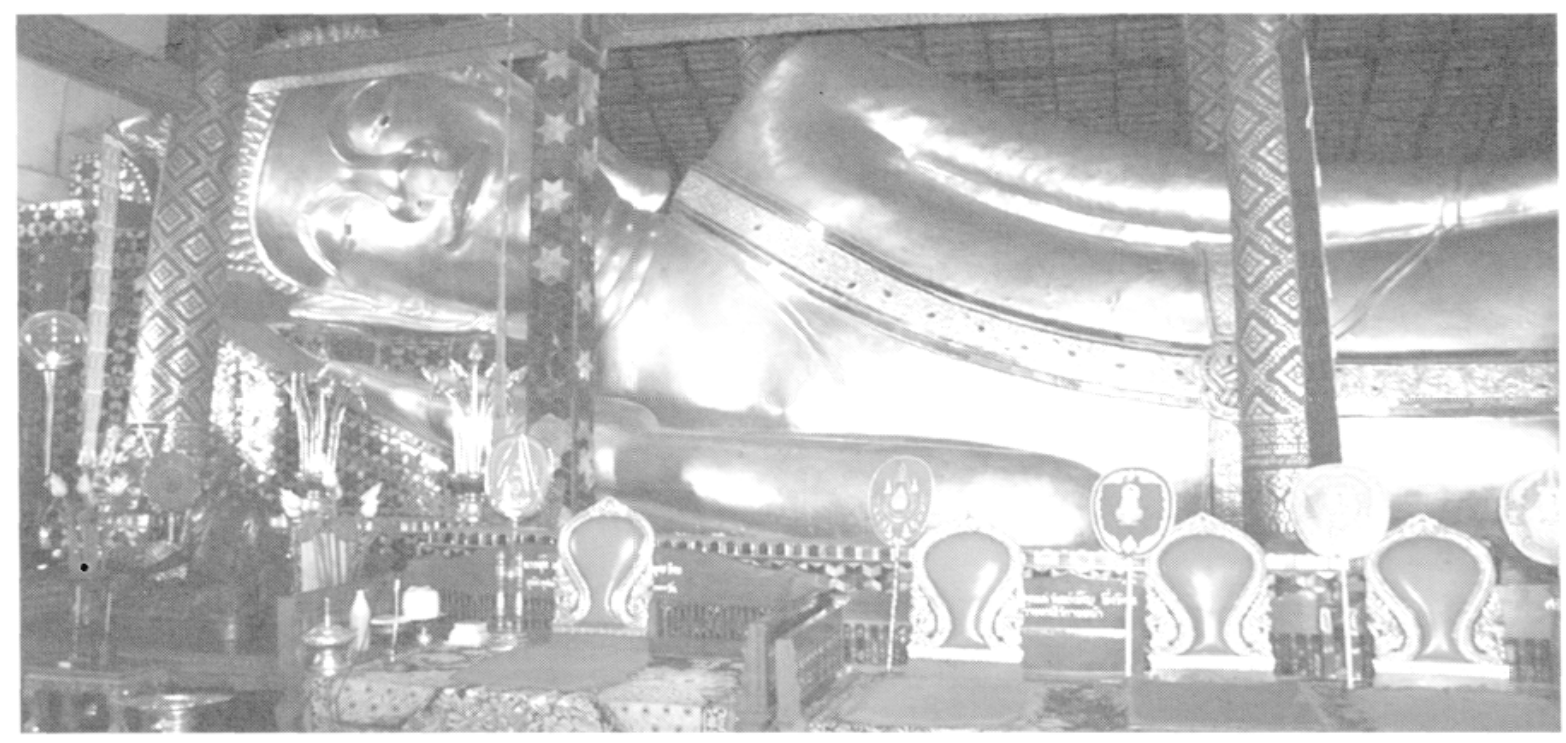

8 - Le Bouddha couché du Wat Phra Non Nong Phueng (ph. F. Lagirarde, novembre 2004)

et le cours des événements ${ }^{18}$. Prasert donne l'explication de Maha Muen selon laquelle la construction d'un canal fut entreprise pour détourner la rivière (Mae Nam Ping) qui coulait dans les environs, du Nord au Sud, Sud-Est. Une première tentative aurait été infructueuse et la réussite ne vint que lorsque la statue d'un Bouddha couché fut installée au monastère qui prit le nom de Wat Phra Non (fig. 8). Alors la rivière se mit à couler dans la direction souhaitée [49].

Le texte nous replace ici au cœur du problème qui s'est posé pour Wiang Kum Kam construite dans une zone inondable et qui d'ailleurs disparut pour cela. Saratsawadi Ongsakun soutient l'idée que la ou les inondations qui sapèrent la ville avant de la recouvrir de sédiments (jusqu'à $1,80 \mathrm{~m}$ par endroits) eurent lieu entre 1558 et 1774 (Saratsawadi 2537, 83) provoquant la "désintégration" des communautés. Selon cet auteur, ce n'est cependant qu'après 1774 que le cours de la Mae Nam Ping changea complètement pour éviter Wiang Kum Kam (id., 84). Alors qu'elle passait à l'Est de Kum Kam pour arriver à Lamphun, aujourd'hui la rivière passe à l'Ouest de l'ancienne cité sans plus jamais atteindre Lamphun (id., cartes p. 21 et 70). La rivière aurait peut-être pu occuper plusieurs lits, d'Ouest en Est, avant de se stabiliser dans celui qu'on observe aujourd'hui: dans ce cas Wiang Kum Kam aurait été construite au point de séparation de ces bras,

18. Ce passage de $\mathrm{KNH}$ se fait l'écho d'un thème bouddhique assèz courant selon lequel le Bouddha, ayant la maîtrise du flot des existences qui l'ont conduit jusqu'à cet état de perfection, contrôle matériellement ce symbole de l'impermanence qu'est l'eau vive des fleuves et rivières. Certains textes nous montrent son disciple Gavampati écartant les eaux, soit pour lui faire passer le Gange, soit pour écarter une crue. 
entourée d'eau à l'Ouest, au Nord et à l'Est. Le poète visitant le Wat Phra Non (qui se trouve aujourd'hui le long de l'ancienne route Chiang MaiLamphun plantée des grands arbres que l'on connaît) est donc tout près de la rivière dans son ancien lit (appelé Ping Hang). Ce que l'on apprend d'important dans KNH c'est que des efforts avaient donc été accomplis (dès 1517?) pour infléchir le cours des eaux (ou pour se protéger de celui-ci?) en aval de Kum Kam. La ville avait-elle été déjà menacée par les inondations avant la grande crue du XVIII ${ }^{e}$ siècle, le détournement de la Ping a-t-il été partiellement une entreprise humaine?

Comme pour illustrer cette théorie le poète signale dans la stance suivante qu'il y a très peu d'eau à cet endroit, pas assez pour que la navigation y soit possible. Le moins qu'on puisse dire est que cet ensemble d'informations très brèves et très allusives venues de KNH semble assez difficile à intégrer dans les données historiques et géographiques qui font autorité sur la région de Chiang Mai.

Après avoir dépassé le Wat Phra Non Nong Phueng le poète se retrouve dans un endroit planté de ton sai (des banians ou Ficus bengalensis). Il prie les esprits des arbres (phra sai chao mai, brah Jrai $\mathrm{caO}_{2} \mathrm{mai}_{2}$ ) de bien vouloir se rendre compte dans quel état de souffrance (dukkha dūjet) il se trouve alors [54].

Le poète continue son voyage faisant diverses remarques sur son environnement naturel. Il atteint une porte (sortie-entrée?) puis emprunte un chemin boueux sur une digue ou une simple levée de terre (khuean) retenant les eaux d'un canal d'irrigation ou de la rivière [64]. Des deux côtés du chemin, il observe alors de nombreux monastères très délabrés ou ruinés (plus d'une centaine, dit-il) qui, de leur temps, devaient être splendides. C'étaient là des lieux où les moines bouddhistes (brah $\mathrm{caO}_{2}$ ) pouvaient pratiquer la méditation "iriy $\bar{a}$ ", c'est-à-dire dans les quatre positions possibles (en marchant, en étant debout, assis ou couché) [65]. Ce passage est étrange et semble impliquer que le poète se trouve dans une zone autrefois prospère mais désormais livrée à l'abandon. Le nombre, certes exagéré, de monastères et leur situation sur le terrain (entre le Wat Non Nong Phoeng et le Wat Yang Num [Wat Kong Sai] que le poète mentionne juste après, il n'y a guère plus de deux kilomètres) nous font douter de la possibilité de cette concentration de bâtiments que l'archéologie ne confirme pas. Si nous prenions cette déclaration au sérieux il faudrait ensuite expliquer pourquoi ces ruines, pourquoi cet abandon. La date de 1517 ne favorise pas l'hypothèse d'une telle décrépitude religieuse. Mais c'est bien là pourtant un thème récurrent dans $\mathrm{KNH}$. Ces ruines sont-elles celles de Kum Kam que le poète signalerait au mauvais endroit (mais Kum Kam était-elle déjà abandonnée)? Ou encore d'autres sites qui nous seraient inconnus? Des inondations les auraient-ils détruits 
ou cette destruction aurait-elle des causes humaines? Il est encore possible que l'identification du Wat Phra Non soit erronée et qu'il faille chercher un autre Bouddha couché dans les ruines de Kum Kam.

Ayant dépassé Kum Kam, le poète se trouve alors dans les environs de l'actuel amphoe Saraphi, un district plus près de Chiang Mai que de Lamphun. Il note donc la présence du Wat Yang Num, mais il est toujours en proie au tourment tandis qu'il arrive dans une zone boisée. Il a une pensée compatissante pour les esprits des arbres (rukkha debată) obligés de s'enfuir lorsque les taillis brûlent [68].

Plus loin dans le texte [73] le poète évoque un nouveau lieu, Hua Fai et une rivière "Ping Kao" (ping kao $\mathrm{K}_{1}$ ). Prasert a voulu comprendre ici un barrage ou un pont sur l'ancienne Ping. Interprétation problématique puisque l'ancien cours de la Mae Nam Ping (à l'Est) appelé aujourd'hui Ping Hang ne pouvait être "ancien" à cette époque (c'était son cours normal). Saratsawadi propose une autre interprétation. Huai Fai serait le nom d'un monastère placé à proximité d'un des bras mineurs de la Mae Nam Ping appelé Ping Kao (fig. 9). Le lieu-dit (Hua Fai) et le bras (Ping Kao) existent toujours sous ces noms. Pourtant on ne peut s'empêcher de souligner que ce bras secondaire (et sous-secondaire comme le Ping Noi) émane aujourd'hui directement ou indirectement du cours moderne de la Mae Nam Ping comme le montre la carte ${ }^{19}$. On peut certes penser que le réseau hydrographique de cette région a connu des bouleversements tels

\section{9 - La paisible Ping Kao en amont du Wat Hua Fai}

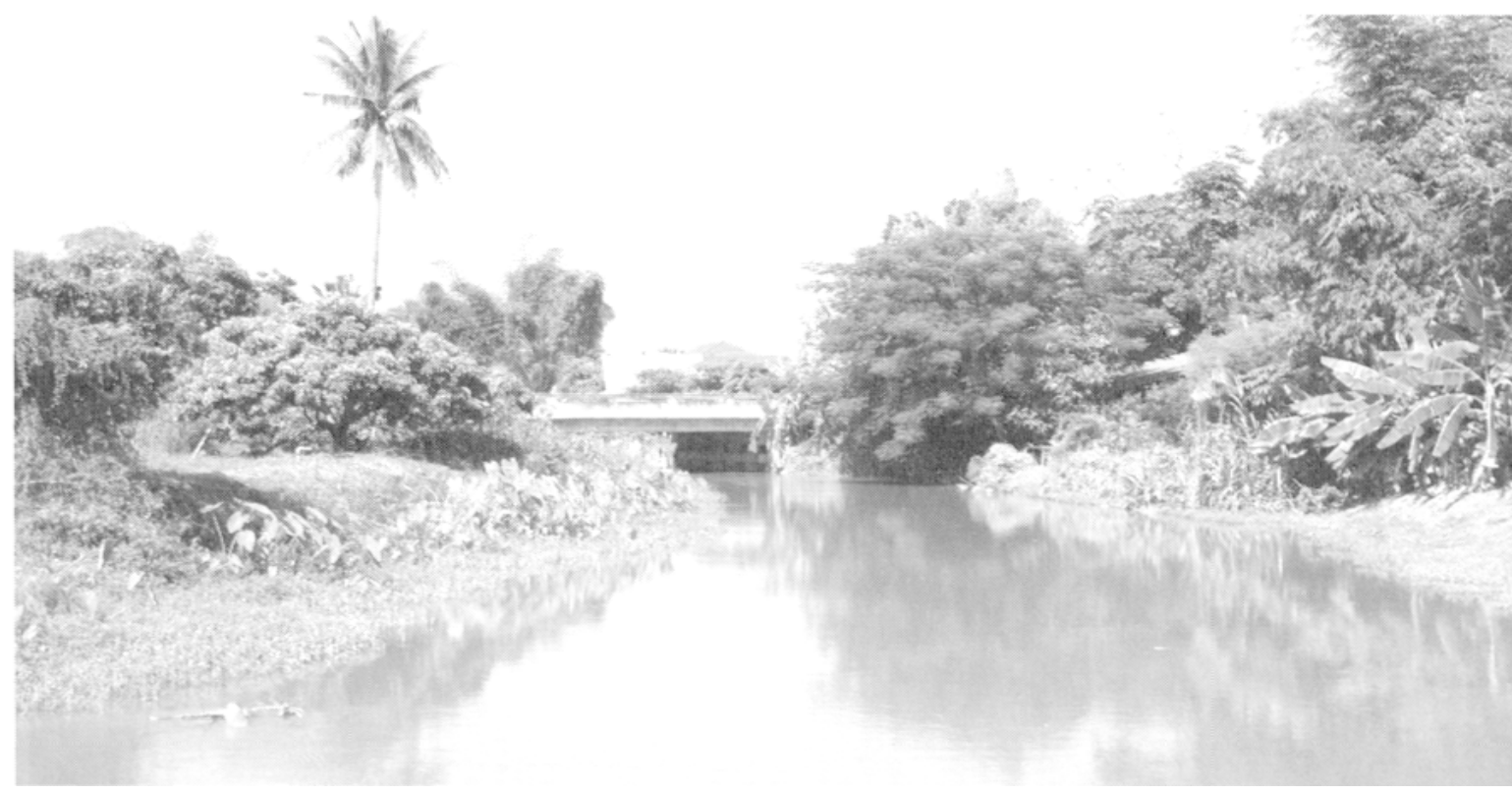

(ph. F. Lagirarde, novembre 2004)

19. Réalisée à partir des cartes au $50000^{\complement}$ du ministère de la Défense. 
que la Ping Kao pouvait peut-être arriver du Nord, le long du chemin que nous attribuons au poète, voire du Nord-Est, au Sud de l'actuelle Saraphi. Mais dans ce cas comment expliquer qu'il y ait à nouveau suffisamment d'eau pour permettre la navigation comme l'explique la strophe suivante? Penth $(1994 \mathrm{~b}, 84)$ affirme que "Kum Kam was located [...] at a spot where the river forked into two branches", ce qui signifierait que le cours actuel de la Mae Ping, contrairement à la carte de Saratsawadi Ongsakun $(2537,21)$, était aussi l'un des cours anciens.

Sur cette rivière (donc la Ping Kao?) le poète remarque en effet que la proue des bateaux porte des fleurs attachées par un ruban de tissu. On y présente également des plateaux garnis d'offrandes [75]. Le texte siamois semble suggérer que le bateau et ses passagers rendent ainsi hommage au Bouddha (jinam). La version de Chiang Mai compare la pratique à une prière que le poète adresserait à sa bien-aimée. Mais il ne s'agit pas ici d'une minuscule barque isolée car, à la strophe suivante, le poète signale sur ces eaux une grande quantité de bateaux et de rameurs [76] plaisante vision qui, par contraste, le rend si malheureux qu'il essuie ses larmes. Bref, il décrit un lieu où un véritable trafic était organisé sur une surface navigable bien nette: ce que n'est plus vraiment la Ping Kao d'aujourd'hui.

Le poète va passer la nuit un peu plus loin, en des lieux peut-être identifiables au Talat Ton Sai (le nom se prononce ton ai en thaï du Nord) dans l'actuel tambon de Hua Fai, amphoe de Saraphi. À ce point se termine la première journée du "voyage" et on se rend compte que notre pèlerin a progressé d'une dizaine de kilomètres. Cette première étape donne bien le ton du poème, à la fois précis et évanescent, un mélange de réalisme et d'artifice qui attire et déstabilise l'analyse. La seule cohérence absolue est dans la plainte monotone du poète qui va continuer de penser à sa bien-aimée dans cette nuit solitaire. Il la compare à un ange au service du dieu Indra [78] tandis que lui demeure dans une situation difficile avec le sentiment que son propre esprit (khwan, khvăñ) pourrait voler en éclats à tout instant [79].

Le lendemain le poète trouve sur sa route un monastère désert (suñ̃̃āāam) et, dans un de ses bâtiments, il observe des peintures murales qui représentent des soldats de différentes origines porteurs de sabres et de boucliers. Il distingue des Thaïs, des Meng (Môns), des Man (Birmans) et des Yiao (Ngiao, Tai Yai ou Shan) [88]. Comme une réponse symbolique à ce thème guerrier, il note l'efflorescence rouge et orange des arbres dits "asoka" (Saraca indica) [89] qui ont poussé autour du monastère. Une nouvelle fois le poète évoque, sans donner de précisions, le souvenir d'événements violents dont nous ne comprenons pas la nature.

Il atteint ensuite un sala où moines et laïcs peuvent se réunir et "prêcher" (thet, desana) puis note justement la présence de moines rassemblés faisant 
remarquer qu'ils sont thaïs (thai china phut, dai jina putra), une précision ethnique assez inattendue [94]. Faut-il la comprendre en opposition à un autre groupe possible? Les Môns ${ }^{20}$ ?

Enfin le poète arrive dans la ville fortifiée de Hariphunchai. Il nous rappelle qu'elle fut fondée avant l'époque du roi Mangrai par un ascète brahmanique (ṛ̂ī) qui en traça le contour sur le sol. Ce rșī avec ses cheveux longs en chignon pouvait voler dans les airs grâce à ses yantras magiques (vedyān). Le poète fait appel à son esprit afin qu'il lui apporte toute l'aide nécessaire pour soulager sa peine et favoriser la réunion ultime avec la femme aimée [103].

Le but du pèlerinage, le reliquaire du roi des rois, apparaît (phra that cao chom chakraphat, brah dhātu $\mathrm{CaO}_{2}$ càm cakka [ou cakrabarti, cakkavati]). Le poète se prosterne [104]. Le "cetiya de la grande relique du Jina" (fig. 10) ressemble à un lingot d'or surmonté d'un parasol d'or à plusieurs étages (chatra). Il est décoré de joyaux (manī) ou du joyau de la relique (selon l'interprétation). Ce cetiya peut illuminer le monde entier et briller encore plus haut dans les cieux (amphon, ambare ou ambara) [106]. Un seul endroit peut lui être comparé, c'est le cūlāmaṇi (le cetiya du Ciel des trente-trois, le Tâvatimsa, qui contient cheveux et clavicule du Bouddha) [107]. La relique, ou le reliquaire, est audessus de tous les maîtres (càm cakka), elle porte les dix marques ou qualités (dasa lakkhana) et elle n'appartient (qu'aux dieux?) du Tāvatimsa [108].

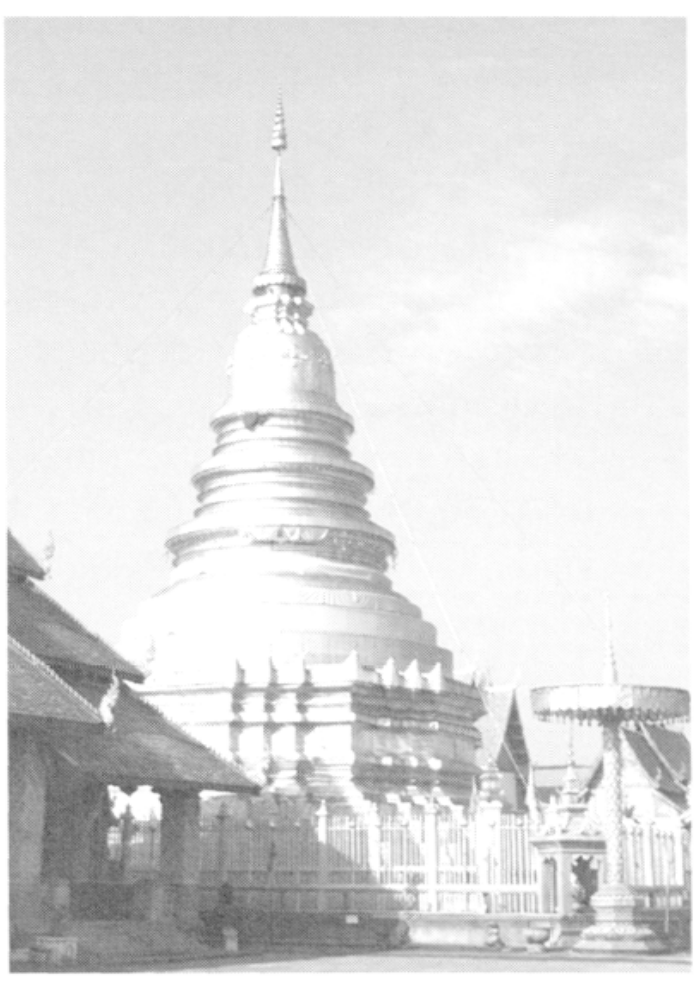

10 - Le grand chedi du Wat Phra That Hariphunchai (ph. F. Lagirarde, novembre 2004)

Le poète fait des offrandes (dăkkhiṇasā), un acte de bucha (pūjā) au bénéfice d'une personne née dans une année précise ici l'année de naissance de sa bien-aimée. Le poète suggère aussi que l'opération pourrait les lier par un charme (sane, sīneha). Il fait peut-être allusion à ces huiles vendues

20. Les ethnonymes caractérisant les Thaïs du Lanna ne sont guère explicites et les intéressés eux-mêmes préfêrent se désigner comme khon mueang, citoyens de telle ou telle ville royale ou principauté. Cependant l'opposition thaïmôn, par exemple, est notée dans le colophon des manuscrits à propos de la dénomination des jours (meng ou thai). 
autour des monastères qui sont censées agir par magie pour attacher un être à un autre [115]. Il vénère également le dieu Indra (Phanta, băntā) qui garde la relique du Bouddha dans sa demeure céleste et qu'il prend à témoin (sakkhī) de sa décision d'offrir de la nourriture, des fruits en particulier, le lendemain matin [116]. Mais, à cet instant, il offre des fleurs d'or (suvannnamālaya) et aussi de la nourriture pour exprimer sa dévotion envers les futurs Bouddhas (Buddhăn kūla) qui protègent le monde. Que la perfection (barami, pārami) du ou de ces Bouddhas vienne protéger la femme aimée [117]! Durant ces dévotions la pleine lune s'est levée et illumine la scène presque comme en plein jour [118].

Le lendemain le poète découvre un millier de "tablettes votives", sans doute des images moulées du Jina en terre cuite (jina bimba), déposées sur les côtés Nord et Sud (du cetiya?). Il fait des offrandes de riz grillé (sājā) pour que les fruits de cette action (phon, bala) soient dédiés à l'être aimé [121].

Il observe un hall d'assemblée ( hra han, brạ̣ hāra = vihāra) construit au milieu du monastère et dans lequel se tient un magnifique Bouddha debout qui semble reposer sur un palais céleste (vimāna). Mais pourquoi, se demande le poète, l'être aimé est-il absent et donc incapable de partager ses aspirations (paniidhāna) [122]? La seule image de Bouddha debout se tient aujourd'hui dans le Wihan Phra Chao Thanchai (vihāra braḥ $\mathrm{caO}_{2}$ dăna cai) qui se trouve non pas au milieu du monastère mais contre son mur Ouest, côté rue.

À nouveau du riz grillé, des bougies, des lampes, des drapeaux et des fleurs sont offerts pour acquérir des mérites dédiés à la bien-aimée. Le poète aperçoit huit statues de khina (khīnāsava, ceux dont les passions intoxicantes ont été détruites $=$ des arahants) ce qui fait un groupe de neuf avec l'Omniscient (sabbaññū, le Bouddha). Le poète leur présente (prakhen) du riz en implorant leur compassion (karūnā) et en leur demandant d'être les témoins (sakkhī) de son intention de transférer ce mérite à sa dame de cœur [124]. Plus loin il interpelle les neuf corps célestes (les planètes, la lune et le soleil) en souhaitant qu'ils constatent ou acceptent ses bonnes actions (kusala) et lui apportent la bonne fortune dont il a besoin [126].

Le poète prie alors Indra, Brahma, Garuda et le/les Naga d'agréer sa vénération mais de partager en deux son bénéfice (kusala). Il invite ensuite Si Kutta (Srī Gutta?) à consigner ses mérites ${ }^{21}$ (puñña) sans oublier de noter le nom de sa bien-aimée dans ses registres puisqu'elle est, précise-t-il, son ăggajāyā, sa reine [127].

21. Il semblerait qu'il s'agisse ici d'un personnage tenant le registre des bonnes et des mauvaises actions, peut-être comme celui représenté dans certains monastères de Chiang Tung (États shans de Birmanie). 
En ce jour de pleine lune, un wan phra ou "uposatha" le poète va s'abstenir de consommer des plats cuisinés (contenant de la viande) et même du riz, il ne boira bien sûr pas d'alcool et se soumettra aux huit préceptes (sīla) [128]. Il les observera dès le petit matin et tout au long de la journée comme s'il purifiait son existence par une discipline qui va lui apporter une sensation de bien-être (ițțhāram [KNH-LJ] ou ițțhāramaṇa [KNH-PNN]) [129].

Une statue de Katchai (Kaccāy[ana]) avec son nombril (nābhī) protubérant est visible dans le monastère. Il y a à cet endroit un tapis pour s'asseoir (pour Kaccāy? pour les moines? pour les dévots?) ainsi que des coussins pour s'accouder. Le poète lui offre des bougies et des drapeaux (et des lampes dans la version siamoise) [141]. Le Wat Phra That Hariphunchai abrite toujours en effet la plus spectaculaire des statues du "Moine Ventripotent" dans un bâtiment récent situé à l'extérieur de la première enceinte, côté Nord.

Le poète va ensuite vénérer (nop) un Bouddha couché (brah saiyāsana). Il admire cette image ainsi que celle des khīnā représentés autour d'elle ${ }^{22}$. Le Bouddha est ici appelé Phra phut (brah buddha) et Phra chao [142]. Il s'agit peut-être du Bouddha couché (ou de son ancêtre) qui se trouve dans le petit wihan du coin Nord-Ouest, entre le grand chedi et le chedi de Suwanna.

Le poète visite ensuite le mondop (maṇdapa) du Phra chao (appelé bo kuea) et offre des bougies, des drapeaux et des lampes au Māra Vijaya. Tout cela au bénéfice de la bien-aimée tandis que lui-même, précise-t-il, demeure le serviteur du Sage (nuea muni) [143]. On identifie ce bo kuea ou bok kluea au Phra Klap Kluea plus connu sous le nom de Phra Daeng (à cause de ses couleurs rouge et rose) installé aujourd'hui dans un wihan moderne au coin Nord-Ouest du monastère.

Le poète quitte alors le Wat Phra That Hariphunchai pour se rendre au Wat Phra Yuen qui est situé à un kilomètre à l'Est en dehors de la ville. Il désire voir les quatre statues de Bouddha (du grand chedi) continuant à prier pour être délivré de la détresse provoquée par la séparation. Il veut être débarrassé (du poids) de ses actions (kè ${ }_{2}$ kamma) [146]. Il est sans doute surpris de constater alors (comme nous l'avons signalé plus haut) que le monastère est abandonné et que les fameuses statues ne sont plus vénérées. Il se rapproche et leur fait des offrandes pour obtenir des mérites dans l'espoir que ceux-ci atteindront aussi la femme aimée tout en le libérant de son mauvais karma [151]. Il nomme un à un les trois Bouddhas du passé, Kakusandha, Kona (Koṇāgamana), Kassapa et celui de notre ère, 
Gotama (khodom) le fondateur de l'actuelle "religion" (sāsanā). Il les prie de bien vouloir apprécier le "goût" (rasa) de ses offrandes [152], expliquant ensuite que les quatre Bouddhas ont atteint le nirvāna (nibbăna) en traversant de nombreuses vies (samsāra nirodha nibbān). Il proclame enfin sa foi dans la venue lointaine d'un futur Bouddha, brah Āraiya (Āriya Metteyya) qui "descendra" sur ce monde pour guider les êtres vers le nibbāna [153].

Par ailleurs, le poète n'a pas manqué de rappeler que Lamphun est un endroit que le Bouddha (Gotama) a visité: "La ville (nagāra) fut dessinée par un ascète (rșī), le Phra chao vint y trouver le repos et c'est également là que Camāde(vi) vint vivre" [148]. De retour au Wat Phra That Hariphunchai, il s'empresse d'aller vénérer une image du Bouddha (chin phimp, jina bimba) qui le représente au moment de son ultime libération (bimokha) c'est-à-dire au moment d'atteindre le parinibbāna. Autour de lui se trouvent ses disciples (parivāra), certains représentés en train de pleurer [155], d'autres gardant le visage presque sec (mukha hmāt) [156]. Une fois encore le poète signale une image du Bouddha couché entouré de ses disciples et on ne sait s'il s'agit d'une statue différente. Le poète, en général, ne semble pas revenir sur les lieux déjà présentés mais, d'autre part, il est bien improbable qu'il y ait eu deux statues du Bouddha couché dans le même monastère.

Certaines statues du Bouddha (trois, quatre, cinq, six, note le poète) sont directement exposées au soleil. Il en éprouve de la pitié et leur offre des bougies et des lampes dans l'espoir qu'elles le protégeront des dangers (ham phoi phai, hām 2 bhoy bhăy) qui pourraient le menacer [157].

Le poète va passer une seconde et demière nuit à Lamphun, continuant à exprimer sa dévotion. Il rend hommage à phra loket klao (brah lokeśa $\mathrm{klaO}_{2}$, Brahma ou Bouddha?) et consacre toujours plus d'offrandes à mettre au crédit de sa bien-aimée. Il vénère encore une fois la relique du Phra chao en espérant qu'il pourra retrouver sa tranquillité d'esprit (tang sati, tăn sati) [158]. Avant l'aube, incapable de dormir, il continue à rendre hommage à un ou des phra (brah) mais il est impossible de savoir s'il s'agit d'une image du Bouddha ou des bons moines du monastère [167]. À nouveau il fait des mérites en insistant solennellement sur le fait qu'il demande leur transfert (on) et expliquant que c'est la raison pour laquelle il verse de l'eau sur le sol [168]. Il prend enfin congé du grand reliquaire où est conservé un os (ațthi) du Jina et fait un ultime sacrifice de riz, de nourriture et de médicaments (?) (khila, gilāna) il ne souhaite rien d'autre en retour que de pouvoir enfin épouser celle qu'il aime [169].

Il quitte la plus belle statue du Jina (varajețtha jina) qui puisse être trouvée dans la ville (de Lamphun) et dans ses environs ${ }^{23}$. Il ne demande 
plus rien sinon que le sort lui évite d'être séparé de son aimée dans une vie ou une autre. Qu'ils soient enfin réunis et puissent connaître ensemble le nirvāṇa [170]! Les dernières bougies, fleurs et lampes sont offertes à la relique du Bouddha: le poète désire un seul être et non pas tous les trésors d'Indra (Bantā) [171].

\section{Le contexte religieux et la dévotion d'un laïc}

Cette longue mais nécessaire paraphrase nous dévoile le vaste paysage mental et ritualiste dans lequel le "pèlerinage" est accompli: la plupart des pratiques mentionnées sont bouddhiques bien que certaines appartiennent à un cadre plutôt brahmanique tandis que d'autres relèvent uniquement du culte des esprits et des divinités des lieux.

$\mathrm{KNH}$ montre sans cesse le poète/héros justifier de gestes ou d'attitudes dévotionnelles par différentes idées ou croyances et par une constante nécessité: s'affranchir de la douleur (frustration-privation) "dukkha" en atténuant la vulnérabilité de l'humain. La dukkha est ici ressentie par l'absence d'un être aimé, un mal d'amour qui deviendrait insupportable si le poète ne le combattait pas par ses actes religieux. Contradiction flagrante puisque c'est précisément le pèlerinage en tant que déplacement qui provoque la séparation. Mais la situation est une donnée du genre et, une fois admise telle quelle, il n'y a donc aucune absurdité: il est vrai par ailleurs qu'on ne connaît pas de cause profonde, "réelle" à cette séparation (si le poète n'explique pas ce point c'est parce qu'il est institué une fois pour toutes hors du récit). Pour se rendre moins vulnérable - mais c'est bien l'idée d'invulnérabilité qui se découvre ici - le poète ne cesse d'implorer la protection qui pourrait émaner de différentes sources de pouvoirs. Il vise aussi le futur, tant proche que très lointain, en accomplissant ses actions méritoires: il réunit volontiers dans celui-ci sa quête amoureuse et son aspiration au nirvāṇa.

Il est parfois difficile de traduire les expressions qui disent la dévotion du poète envers les images du Bouddha ou d'autres êtres "surnaturels" bien moins importants. En général elles signifient marquer le respect par un geste particulier. La première de toutes est le wai $\left(\right.$ hvai $_{2}$ ) qui consiste à joindre les deux mains à hauteur du visage en s'inclinant plus ou moins. Mais il faut en signaler bien d'autres: kraphum ou phanom mue (krahbum $_{(1)}$, banam mị) [13] namatsakan (namassakāra) [17], nop (nàp, nap) [19], pranom nom na (Prah hnam nàm ${ }_{2}$ hnā $\bar{a}_{2}$ ) [16 KNH-LJ]. Ces gestes de salut avec une inclination ou une prosternation sont complétés par des gestes d'offrande thawai (dhavāy) prakhen (prahgen) [124] d'objets rituels (bougies, petits drapeaux de tissu ou papier, lampes), de fleurs, de nourriture, de différentes préparations ou sortes de riz et de médicaments. 
Toutes ces actions propices sont "karmiquement bonnes" (kuson, kusala) et génèrent donc du mérite (puñna), un capital qui peut être transmis au profit d'une autre personne que celle ayant agi. Ce transfert se dit on (oàn) en siamois (peut-être du verbe pāli "onojeti", dédier, distribuer) et oy ou owai (oàvaya) en thaï du Nord [11 KNH-LJ]. Ce don est matériellement exécuté par le rituel dit kruat nam qui consiste à verser doucement de l'eau gardée dans un récipient directement sur le sol. D'habitude la "libation" se fait à l'intérieur du monastère, éventuellement au pied d'un arbre de la Bodhi. Cette prise de la terre à témoin, souvent accompagnée par une récitation de formules par les moines du lieu, reprend des éléments de l'épisode de l'Éveil de Gotama à usage laïque mais pourrait être d'origine animiste (Zago 1972, p. 127-128 et notes 118 à 122). La cérémonie de transfert est dédiée généralement aux défunts (d'ordinaire les parents) puisque ce sont eux qui en ont le plus besoin, mais dans $\mathrm{KNH}$ ce n'est bien entendu jamais le cas.

Un autre acte qui se conforme aux enseignements bouddhiques est le respect - la pratique - des préceptes moraux. Le poète mentionne les "beñcā" (c'est-à-dire pañca, les cinq, les cinq préceptes ou "moralités" de base appelés sīla) une première fois [11] en expliquant que leur pratique régulière apporte une réelle protection contre l'anxiété (tam ngon, tām ${ }_{1}$ nan) et, par conséquence, le bonheur dans l'existence. Il mentionne ensuite les huit préceptes [128] dont la pratique convient mieux à une personne effectuant un pèlerinage et qui séjourne à l'intérieur d'un monastère (en addition aux cinq moralités de base, trois nouvelles règles doivent être suivies: s'abstenir de prendre de la nourriture en dehors des heures prescrites, c'est-à-dire dans l'après-midi, s'abstenir de spectacles de danse, de musique, de bijoux et de parures, s'abstenir de siège ou de couche confortable ${ }^{24}$ ). Comme pour le kruat nam il existe une cérémonie accomplie par les moines du monastère qui, à la demande des dévots, "invitent" et donnent les préceptes. Mais $\mathrm{KNH}$, qui reste généralement muet sur les rapports entre religieux et laïcs, ne dit pas si notre poète les a reçus de cette façon.

La dévotion envers les images de Bouddha, sa relique et son reliquaire est bien entendu le point fort du poème: ce sont vers ces buddhacetiya, "monuments" ou objets commémoratifs ${ }^{25}$ que se dirige naturellement la dévotion bien que théoriquement elle puisse également se tourner vers d'autres objets, les dhammacetiya par exemple, objets sur lesquels est inscrite la doctrine bouddhique, le Dhamma, (ce sont donc des citations de vers canoniques ou de commentaires). Les images du Bouddha sont clairement reconnues et nommées. Le poète utilise de plus un grand choix

24. Cette liste, qui suit celle de Wells $(1975,53)$, est un peu différente de celle donnée par Zago $(1972,114)$.

25. Dans le sens défini par le prince Damrong Rajanubhab et A. B. Griswold dans l'ouvrage Monuments of the Buddha in Siam (1973, V-VI et 9-10). 
d'épithètes du Bouddha (jina, jina uḷara, jina Māra, sabbaññu, brah sețthī, sayambhū, jețțha, Māra vijaya, brah bodhi ñāna). Phra phut (braḥ Buddha) n'est donné qu'une seule fois de même que Khodom (Gotama).

La sixième strophe du poème rappelle que les quatre Omniscients (trois Bouddhas du passé et le Bouddha de notre ère) ont disparu définitivement mais que le monde espère toujours la venue de Metteyya (Āraiya). Ceci n'est cependant qu'une remarque théorique et le poète ne se réfère en l'occurrence à aucune image rencontrée sur sa route ni à aucun culte particulier. En revanche, plus loin, le poète nomme ces trois Bouddhas du passé lorsqu'il visite le Wat Phra Yuen [151] leur accordant alors les mêmes honneurs que ceux qu'il prodigue au Bouddha Gotama.

Le culte probable des huit arahants disciples du Bouddha, évoqué à la strophe 124, est une autre caractéristique importante de $\mathrm{KNH}$. Bien qu'ils ne soient pas nommés personnellement, ces disciples sont présentés comme les paet khina (les 8 khinnāsava, "ceux en qui tous les débordements ont disparu" = les arahants) et le poète n'oublie pas de préciser que leur groupe n'est complet qu'avec le neuvième et suprême arahant, le Bouddha lui-même. On ne peut donc que faire le lien avec la tradition écrite et le culte des arahants que nous connaissons par ailleurs. Ce groupe est toujours symbolisé par le maṇdala des huit disciples, un diagramme bien connu qui correspond aussi au plan en "profondeur" de certains bâtiments monastiques, les halls ou salles d'ordination en particulier. Ces édifices sont dessinés sur un espace en huit points, chaque point correspondant à un disciple et matérialisé par une boule de pierre (luknimit) enfouie dans la terre comme la graine ou racine de la feuille de siman, la borne qui marque les limites dans lesquelles doivent se tenir les actes officiels de la Communauté monastique. Certaines de ces bornes sont d'ailleurs sculptées de l'image des disciples. Au cœur de l'édifice une neuvième boule de pierre représente le Bouddha. Le diagramme apparaît ainsi:

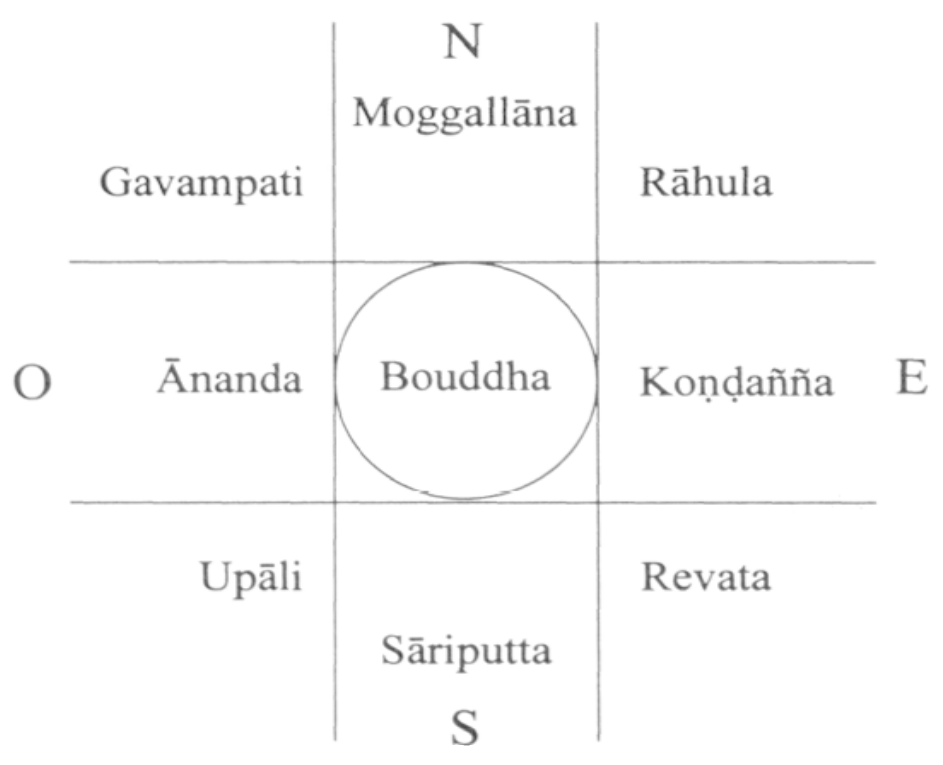


Cette disposition rituelle est décrite, sinon expliquée, dans une partie du texte appelé Mahädibbamanta appartenant au genre des paritta non canoniques, très connu dans le bouddhisme indochinois. On trouve également ce texte sous le titre Cülasabbadisabuddhamangalaparitta.

L'élément peut-être le plus surprenant, dans $\mathrm{KNH}$, réside dans la mention du culte de Kaccāy sans aucun doute la plus ancienne et la plus claire qui soit. Kaccāyana, le moine ventripotent également connu depuis le début de l'époque Ratanakosin sous le nom de Sangkachai, est une personnalité incontournable - car des plus visibles - du "panthéon" thaï. Son image typique est installée dans tous les bâtiments ordinaires des monastères, voire dans des wihan particuliers. On ne connaît pas, cependant, disons à cette période $\mathrm{du} \mathrm{XVI}{ }^{\mathrm{e}}$ voire du XVII ${ }^{\mathrm{e}}$ siècle, d'autres documents citant ce nom de Kaccāy, en évoquant son aspect de moine obèse et le culte qui lui était rendu. Il semble qu'à une époque antérieure chez les Môns, les Birmans et les Thaiis du Nord, seul le nom de Gavampati ait été utilisé pour la même personne: c'est le cas par exemple dans la chronique du Wat $\mathrm{Pa}$ Daeng qui rappelle les problèmes réels ou imaginaires autour du culte de Pha Gawam (brah Gavampati) ${ }^{26}$. Il n'est pas indifférent que cette image soit installée dans le bastion de la souche mône du bouddhisme du Lanna ${ }^{27}$ : c'est le rappel de son origine et de son développement dans une autre culture d'accueil.

Il est toujours possible de voir cette image de Kaccāyana ${ }^{28}$ dans le Wat Phra That Hariphunchai: si elle est aujourd'hui abritée dans un wihan de type siamois, ce ne fut pas toujours le cas et des photographies anciennes de Lamphun montrent qu'autrefois le moine se tenait à l'air libre (Lamoon 2532, fig. 53). Cependant s'il faut comprendre que tapis et coussins notés dans KNH se tenaient autour de lui - pour les moines et dévots? - on pourrait penser qu'il existait alors un toit abritant la statue et ces accessoires. À moins que le texte n'évoque des tapis et des coussins sculptés avec le moine.

Le poète fait également état du culte des amulettes, une autre grande caractéristique du bouddhisme indochinois, à la strophe 121. Ces amulettes

\footnotetext{
26. Ce point de dispute très particulier se retrouve dans la chronique du Wat $\mathrm{Pa}$ Daeng de Chiang Tung, traduite par Sao Saimöng Mangrai (1981, 135), ainsi que dans le Tamnan wat pa daeng de Chiang Mai, édité par Bamphen Rawin $(2538,40)$.

27. Pour une discussion générale sur l'origine et le développement du culte de Gavampati-Kaccāyana entre mondes môns, birmans et thaïs, voir mes deux articles publiés sur le sujet (Lagirarde 2000 et 2003).

28. C'est une imposante masse de briques stuquées qui a sans doute subi de nombreuses restaurations. On la trouve à l'extérieur de la première enceinte du wat, du côté Nord.
} 
qu'il décrit en utilisant l'expression chin phimp (jina bimba) sont sans doute des petites plaquettes de terre moulées et cuites reproduisant une image du Bouddha qui étaient soit déposées par les pèlerins autour du cetiya (ici KNH dit sur les côtés Nord et Sud, sans préciser) soit disponibles moyennant une petite donation. Elles pouvaient ainsi être placées comme offrandes ou encore, on l'imagine, rapportées après le pèlerinage pour être vénérées chez soi.

En dehors de ces éléments relevant essentiellement du culte bouddhique - et qui nous en montrent la continuité car rien ne semble différent d'aujourd'hui - KNH renvoie plus largement à la culture indienne en faisant parfois référence à des sujets que le bouddhisme partage (notamment avec le brahmanisme). Le culte des ermites (rșī) est de ceux-là: présentés comme des précurseurs ou des annonciateurs du Bouddha, leur fonction est toujours liée à une appropriation symbolique des lieux susceptibles de se développer en principautés ou royaumes reconnaissant le Dharma. L'ermite aux cheveux longs en chignon qui fonda jadis Hariphunchai pouvait voler dans les airs grâce à sa connaissance du Veda (sans doute pris dans le sens de doctrines magiques) matérialisée par des yantras magiques (vedyān) mais son esprit, ses pouvoirs sont toujours communicables et peuvent être invoqués par ceux qui désirent recevoir une aide directe. Aujourd'hui encore, on rend volontiers dans les monastères un culte à ces ermites ${ }^{29}$ mais ce sont également des esprits avec lesquels les médiums communiquent. $\mathrm{KNH}$ dans cette séquence très concise (ermite-Bouddha-princesse "locale" [148]) n'en donne pas moins la structure fondamentale de la préhistoire mythologique commune en pays thaï: tous les acteurs (le quatrième étant le souverain thaï) sont présents et demeurent présents dans un au-delà auquel chacun croit. Signalons que ces yan (yān) dessinés ou imprimés sur des morceaux d'étoffe font toujours partie des accessoires religieux de base des bouddhistes de Thaïlande.

Dans les strophes qui se passent à Chiang Mai, on a bien sûr remarqué le respect du poète pour l'esprit du roi Mangrai, lequel mourut exactement deux cents ans (1317) avant la date supposée de ce pèlerinage, et pour sa reine. La vénération pour les lieux et leurs maîtres s'expose ainsi par une structure à quatre temps (préparation par l'ermite, sacralisation par le Bouddha, reconnaissance de l'autochtonie des populations et prise de pouvoir définitif par le souverain) qui est le schéma typique de la légitimation de la situation historique des Thaïs. Celui-ci se retrouve dans toutes les chroniques traditionnelles et $\mathrm{KNH}$ n'échappe pas à ce modèle.

29. On retrouve aussi bien leur image au Wat Chedi Luang qu'au Wat Phra That Doi Kham à Chiang Mai, pour ne citer que deux lieux parmi les plus connus. 
Pour en finir avec ce panthéon mesuré, toujours intégré sans rejet au bouddhisme thaï, signalons en passant les ogres ou démons (yakșa) les esprits des portes ou des seuils (mahesak sat) ainsi que ceux des arbres et des eaux. Indra demeure apparemment le dieu préféré (parfois présenté sous le nom de Bantā) mais Brahma, Garuda et Nāga sont aussi mentionnés. Le poète fait par ailleur allusion au pouvoir du khwan ("l'esprit gardien" de la personne) ainsi probablement qu'aux charmes et potions magiques (nam man phray) qui sont des produits toujours disponibles dans les talat phra (marché aux amulettes) de la Thaïlande.

\section{Une excursion historique}

ou une promenade imaginaire (mais bien documentée) dans la mythologie et l'histoire thaïes?

La valeur historique directe de KNH n'a jamais été remise en question. Par "directe" il faut entendre une expérience unique réalisée par un seul et même individu dans une unité de temps et de lieu précis.

En assignant à cette expérience la date de 1517 - date imaginable à l'intérieur du système fictionnel lui-même (ce qu'est par définition une poésie) - les savants lecteurs de KNH se sont enfermés dans un cadre problématique où il est difficile de faire entrer tous les éléments descriptifs du texte. Il est à craindre que cette date soit en cours d'adoption définitive par les historiens thaïlandais qui pourront ainsi dater des vestiges historiques sur lesquels les méthodes d'analyse archéologique ne se prononcent pas clairement (par exemple: si le poète passe par une seconde porte après la porte de Chiang Mai c'est parce qu'il y a une seconde enceinte, la levée de terre appelée kamphaeng din, qui est donc antérieure à $1517^{30}$, etc.). Cette date est fondée sur des indications de valeur contestable: une année cyclique et la présence du Bouddha d'Émeraude à Chiang Mai. Les années cycliques (dans $\mathrm{KNH}$ sans mention de date en chiffre d'une ère quelconque qui viendrait la confirmer) sont souvent faussement attribuées dans les manuscrits tandis qu'il est difficile de se faire une opinion exacte sur le ou les Bouddhas d'Émeraude qui circulèrent au Lanna.

Car l'archéologie, l'histoire et l'anthropologie bouddhiques, sans nier la valeur du texte qui demeurera quoi qu'il en soit le meilleur panorama d'une expérience religieuse vécue dans un passé indéfini, mais relativement lointain, ne peuvent que souligner les zones d'ombre, les mystères ou les

30. Les deux rapports de fouilles publiés par le département des Beaux-Arts de Chiang Mai (Krom Silapakon 2540 et 2545) ne donnent aucune réponse définitive sur le début de la construction du kamphaeng din que le poète semble traverser. 
contradictions qui l'habitent. Certes on ne peut reprocher à un texte, qui n'a pas de prétention historiographique, de passer sous silence ce que nous aimerions entendre, pourtant la lecture de $\mathrm{KNH}$ montre bien que le poète a tenté et assez bien réussi l'écriture d'un journal de voyage qui cherche à décrire non seulement les hauts lieux de la pratique religieuse entre Chiang Mai et Lamphun mais aussi les voies qui y conduisent. Mais ce document, somme toute réaliste, nous échappe largement sur cinq thèmes:

1 - La Communauté des moines, son identité et ses rapports avec la communauté laïque;

2 - L'abandon de monastères;

3 - Wiang Kum Kam et l'état de sa communauté;

4 - Le réseau hydrographique de la Mae Ping et de ses bras;

5 - La seconde porte et la présence de la seconde enceinte de Chiang Mai.

Sans vouloir reprendre la liste des questions relatives à ces cinq points nous pouvons néanmoins commenter à nouveau les deux premiers puisqu'ils touchent précisément à l'histoire religieuse. Comme on le sait, la vie des lieux de culte dépend de l'appui qu'ils reçoivent: que celui-ci vienne à manquer pour une raison quelconque et le sanctuaire est mort. Alors que beaucoup d'épigraphes nous apprennent précisément qui soutenait tel ou tel monastère, quels étaient les noms des esclaves-serviteurs qui y étaient assignés voire qui était le religieux qui en avait la charge, on remarque que $\mathrm{KNH}$ exclut presque systématiquement toute référence à des personnages quelconques, à des groupes voire à des fonctions précises. Aucune rencontre directe avec qui que ce soit n'est racontée et l'absence des grands responsables du fonctionnement des institutions décrites (la ou les principautés, les villes, les monastères, la religion) est frappante. En ce sens $\mathrm{KNH}$ est également bien différent des chroniques dont le but est souvent de fixer dans la mémoire des noms, des titres et des généalogies. JKM sur la même période et les mêmes lieux, fournit des quantités de détails montrant en particulier les trois différents groupes de bhikkhus toujours en présence (le nikāya "traditionnel" et les deux groupes "cinghalais" qui forment un tigana). Plus curieusement encore les rituels bouddhiques qui mettent en relation les laïcs avec la communauté monastique sont complètement ignorés. On a vu par exemple que deux cérémonies décrites dans $\mathrm{KNH}$ (transfert des mérites et prises des préceptes) se passaient de la direction d'un moine mais il est encore plus stupéfiant de se rendre compte que le seul rituel véritablement incontournable dans la pratique bouddhique et la quête des mérites, l'offrande de nourriture matinale aux moines, n'est sinon jamais accompli, du moins jamais décrit, même de la façon très allusive avec laquelle procède généralement le poème. Un seul passage de $\mathrm{KNH}$ signale des religieux; ce sont ces thai china phut, ces "fils thaïs 
du Bouddha" notés plus haut [94] dont la caractérisation ethnique (thai) possède quelque chose de troublant et qui ne font qu'apparaître dans un contexte peu précis tandis qu'une dernière allusion à un (des) "phra" ne nous permet pas de savoir s'il s'agit d'un moine ou d'un Bouddha [167].

On est donc en mesure de se poser la question de savoir pourquoi le Sangha est le grand oublié de ce texte. Le poète a-t-il consciemment voulu éviter ce sujet ou bien son mode d'écriture ne s'accommodait-il pas d'autres personnages que la femme aimée? Y avait-il des raisons objectives de passer sous silence la présence des communautés monastiques? On ne comprend guère ce point qui est renforcé par un arrière-plan de détresse assez perceptible (trois sites monastiques abandonnés, le rappel d'événements guerriers).

Là encore on ne peut que souligner les problèmes: pourquoi tant de monastères déserts entre Wiang Kum Kam et Lamphun? S'agit-il d'un abandon de la région provoqué par une catastrophe naturelle ${ }^{31}$, ou par les guerres? Ou d'un manque de moines pour des raisons internes à la Communauté? Rien dans les années qui précèdent cette date de 1517 ne vient vraiment expliquer cette sombre image. Le dernier des monastères abandonnés, selon KNH, est le Wat Phra Yuen. Or, on a bien vu plus haut combien il est improbable que ce monastère, construit en 1370 pour accueillir le célèbre thera Sumana, ait été abandonné 137 ans après alors que la tradition monastique de Sumana (les Pupphavāsī ou bhikkhus du Wat Suan Dok) était toujours forte ${ }^{32}$.

Mais pour l'année qui nous intéresse (1517 ou plus exactement avril 1517 à mars 1518) il n'y a guère dans l'histoire de signal qui indiquerait les problèmes soulevés par KNH. La JKM (Cœdès 1925, 129) fait le rappel de grands et fastueux événements religieux qui mettent en évidence le respect que le roi de Chiang Mai cultivait pour le grand monastère et les moines de Lamphun:

Du huitième jour de la lune croissante de Māgha au jour de la pleine lune [de l'année 879 de la petite ère bouddhique, soit janvier 1618], le roi adora la Grande Relique de Haripuñjaya et écouta la lecture du Buddhavamsa.

31. Les seules inondations dramatiques signalées par $\mathrm{CM}$ sont celles de 15241525. Elles eurent lieu à Chiang Rueak au marché de Si Phum (CM-N, 151) et coûtèrent la vie à un grand nombre de gens. Ces inondations furent causées par la Nam Kha (CM-W, 108). Si Phum se trouve au coin Nord-Est de la cité carrée de Chiang Mai, à l'extérieur des remparts.

32. A. B. Griswold affirme que, selon sa lecture de la Mūlasāsanā, le monastère était habité au XVe siècle et aurait continué de l'être jusqu'en 1595 (1975, 44 et 48).

Il ne dit pas malheureusement sur quelle Mülasāsanā il fonde cette réflexion. 
Puis il entoura la Grande Relique d'un mur en or valant plus de cent mille. Le mercredi, neuvième jour de la lune décroissante du même mois, au moment de la conjonction de la lune avec le nakșatra Visākha, il commença la construction d'un vihāra au Nord (de la Grande Relique).

La Chronique de Chiang Mai nous montre que la population sujette au roi Mueang Kaeo s'était augmentée en 1506, 1508 et 1517, par l'arrivée de nouveaux serfs offerts par des chefs de principautés voisines (CM-N, 146 et 148-149; CM-W, 104-106). Il y avait donc assez de monde pour que les gens de Chiang Mai et des mueang voisins (inévitablement Lamphun) fabriquent ensemble des briques (1516-1517) pour construire ou restaurer les remparts de la ville (1517-1518).

Les pratiques bouddhiques, quant à elles, sont bien homogènes et rien ne les distingue de celles d'aujourd'hui sinon que (mais c'est encore un de ces "silences" qu'on ne saurait reprocher à KNH) certaines caractéristiques birmanes du bouddhisme du Lanna n'apparaissent pas dans le texte: par exemple le culte d'Upagutta (Upakhut) ou du Phra Bua Khem, le Bouddha "au lotus et piqué d'aiguilles". Bref KNH montre tous les points de compatibilité entre la culture religieuse du Lanna et celle du Siam. L'aspect thaï (pan-thaï?) unifiant cimente le texte et aucune allusion aux différentes perceptions d'orthodoxie et d'orthopraxie à l'intérieur de la Communauté monastique du Lanna n'est d'ailleurs reconnaissable.

Il n'est pas impossible que l'auteur de $\mathrm{KNH}$ ait souhaité présenter une image totalement "inoffensive" et non polémique du bouddhisme au Lanna en se concentrant sur les monuments du Bouddha (cetiya au sens déjà donné) et l'effort de dévotion personnelle qu'ils exigent naturellement. Dans ce cas, il lançait une sorte d'appel général à la donation et à des actes constructifs de toute nature pour soutenir la religion unifiée. Il est vrai que la phase "déclin et chute" (Grabowsky 2004, 22) allait débuter juste après le second couronnement de Mueang Kaeo en 1520 et se précipiter après sa mort en 1525. Mais cette image du bouddhisme uniformisé en 1517 est peu satisfaisante et, plutôt que de l'accepter telle quelle, il est probablement plus sage de considérer $\mathrm{KNH}$ comme un texte non datable avec précision mais sans doute plus récent qu'on ne l'a cru.

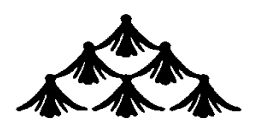




\section{Bibliographie}

Aroonrut WICHIENKEEO

2539a - Photchananukrom sap lanna chapho kham thi prakot nai

[1996a] bailan (The Northern Thai Dictionary of Palm-leaf Manuscripts), Chiang Mai, Suriwong Book Center (Silkworm Books), $797 \mathrm{p}$.

$2539 \mathrm{~b}$ - Wat rang nai wiang chiang mai

[1996b] [Les monastères abandonnés de Chiang Mai], Chiang Mai, Suriwong Book Center, $128 \mathrm{p}$.

CEDÈs, George

1925 - "Documents sur l'histoire religieuse et politique du Laos occidental", BEFEO XXV, p. 1-200 [Ces "Documents" contiennent en particulier une traduction de la Jinakâlamāli (Jinak 4.2.1) texte en pāli composé à Chiang Mai en 1516/1517 et augmenté en 1527 par le moine Ratannapañña du Wat Pa Daeng. Édité par A. P. Buddhadatta, 1969, Londres. La traduction en anglais de ce texte a été publiée par N. A. Jayawickrama, 1968, The Sheaf of the Garlands of the Epochs of the Conqueror, Londres, PTS].

Bamphen RAWIN (éd.)

2538 - Tamnan wat pa daeng [Chronique du Wat Pa Daeng], ekasan

[1995] witchakan ruam somphot 700 pi Chiang Mai andap 5, sathaban wichai sangkhom, mahawithayalai Chiang Mai, 91 p.

Chaiyot ITWORAPHAN et Phanuphong LAOHASOM

2543 - Wihan lai kham wat phra sing: sathapatkam lae chitrakam

[2000] faphanang [Le Wihan Lai Kham du Wat Phra Sing architecture et peintures murales], Chiang Mai, Suriwong Book Center, 111 p.

Damrong RAJANUBHAB (Prince)

1973 - Monuments of the Buddha in Siam, seconde édition révisée, introduction de A. B. Griswold, Bangkok, The Siam Society, 60p.

GEDNEY, William J.

1989 - "Siamese Verse Forms in Historical Perspective", Selected Papers on Comparative Tai Studies, edited by Robert J. Bickner, John Hartmann, Thomas John Hudak and Patcharin Peyasantiwong, Michigan Papers on South and Southeast Asia, Center for South and Southeast Asian Studies, The University of Michigan, Number 29, p. 489-544. 
GRABOWSKY, Volker

2004 - "The Northern Tai Polity of Lan Na (Babai-Dadian) between the Late 13th to Mid-16th Centuries: Internal Dynamics and Relations with Her Neighbours", ARI Working Paper $n^{\circ} 17$, January 2004, www.nus.ari.edu.sg/wps.htm.

GRISWOLD, Alexander B.

1975 - Wat Phra Yün Reconsidered. Bangkok, The Siam Society, monograph $\mathrm{n}^{\circ} 4,88 \mathrm{p}$..

HUDAK, Thomas John

1990 - The Indigenization of Pali Meters in Thai Poetry, Athens, Ohio University Center for International Studies, Southeast Asia Series n ${ }^{\circ}$ 87, 237 p.

Krom Silapakon

2530 - Wannakam samai ayutthaya, lem 2 [La littérature de l'époque

[1987] d'Ayutthaya, volume 2], Krungthep, kong wannakadi prawatsat krom sinlapakon, phim thi borisat amarin printing group chamkat.

2540 - Rai-ngan kankhutkhonsueksa lae burana kamphaeng mueang

[1997] Chiang Mai sanoe thesaban nakhon Chiang Mai [Rapport sur les fouilles entreprises sur les remparts de Chiang Mai, document consultable au département des Beaux-Arts, Musée national, Chiang Mai].

2545 - Rai-ngan kankhuttruat chan din thang borannakhadi kamphaeng

[2002] din pom Hai Ya Tambon Hai Ya Amphoe mueang Chiang Mai changwat Chiang Mai [Rapport sur les fouilles entreprises sur la levée de terre de Chiang Mai, au fort de Hai Ya à Chiang Mai, document consultable au département des Beaux-Arts, Musée national, Chiang Mai].

LAGIRARDE, François

2000 - "Gavampati et la tradition des quatre-vingts disciples du Bouddha: textes et iconographie du Laos et de Thaïlande", BEFEO 87-1, p. 57-78.

2002 - "Notes sur la fondation politique et religieuse du Lanna dans le mythe de Suvanna Khamdaeng", Aséanie 9, p. 80-100.

2003 - "Devotional Diversification in the Thai Monastery: the Worship of the Fat Monk", The Buddhist Monastery: a cross-cultural survey, P. Pichard et F. Lagirarde (éds.), Paris, EFEO, Études thématiques 12 , p. 149-168. 
Lamoon JANHOM (Lamun Chanhom)

2532 - Khlong Nirat Hariphunchai kanvinichai ton chabap (Khlong

[1989] Niras Hariphunchai: a text critical study), thesis submitted for the degree of Master of Art, department of Lanna Language and Literature, Chiang Mai University, $457 \mathrm{p}$.

2538 - Wannakam thongthin lanna (Lanna litterature), Chiang Mai,

[1995] Suriwong Book Centre, 208 p.

Manas CHITAKASEM

1972 - "The emergence and development of the Nirăt genre in Thai poetry", Journal of the Siam Society, vol. 60 part 2, p. 135-168.

PENTH, Hans

1994a - A Brief History of Lān Nā: Civilizations of North Thailand, Chiang Mai, Silkworm Books, 74 p.

$1994 \mathrm{~b}$ - Jinakâlamālì Index, an annotated index to the Thailand part of Ratanapañña's Chronicle Jinakālamālī, Oxford and

Chiang Mai, The Pali Text Society et Silkworm Books, 358 p.

Prasert na NAGARA

2516 - Khlong Nirat Hariphunchai sop kap ton chabap chiang mai

[1973] [Le Khlong Nirat Hariphunchai (de la Bibliothèque nationale) comparé avec un original de Chiang Mai], Bangkok (sans mention d'éditeur), $244 \mathrm{p}$.

Sao Saimöng MANGRAI

1981 - The Pädaeng Chronicle and the Jengtung State Chronicle Translated, Ann Arbor, The University of Michigan, $301 \mathrm{p}$.

Saratsawadi ONGSAKUN (Sarasawadee Ongsakul)

2529 - Pravatisat lanna [Histoire du Lanna], $3^{e}$ édition 2544 [2001],

[1986] Bangkok, Samnak phim Amarin, 524 p.

2537 - Wiang kum kam, kan soeksa prawatsat chumchon boran nai

[1994] lanna [Wiang Kum Kam, Étude de l'histoire d'une ville antique du Lanna], Chiang Mai, Phak wicha prawatsat khana manutsat mahawithayalai chiang mai, $161 \mathrm{p}$.

SCHWEISGUTH, $P$.

1950 - "Les 'Nirat' ou poèmes d'adieu dans la littérature siamoise", Journal of the Siam Society, vol. 38 part 1, p. 67-78.

1951 - Étude sur la littérature siamoise, Paris, Librairie d'Amérique et d'Orient, $409 \mathrm{p}$. 
SWEARER, Donald K.

1976 - Wat Haripuñjaya: A Study of the Royal Temple of the Buddha's Relic, Lamphun, Thailand, Missoula, Montana, Scholars Press.

Udom RUNGRUEANGSI

2542 - "Nirat hariphunchai, khlong" [article "Khlong Nirat

[1999] Hariphunchai"], Saranukrom wattanathamthai phaknuea lem 7 [Encyclopédie de la culture thaie, région du Nord, vol. 7], Krungthep, Mulanithi saranukrom wattanatham thai, thanakhan thaiphanit, p. 3291-3297.

2546 - Wannakam lanna [La littérature du Lanna], Krungthep,

[2003] Samnakngan kongthun sanapsanun kanwichai, 679 p.

WOODWARD, Hiram W. Jr.

1997 - "The Emerald and Sihing Buddhas: Interpretations of their Significance", Living a Life in Accord with Dhamma Papers in Honour of Professor Jean Boisselier on his eightieth Birthday, Eilenberg, Natasha; Diskul, M. C.; Brown, Robert L. (eds.), Bangkok, Silpakorn University, p. 502-513.

WELLS, Kenneth E.

1975 - Thai Buddhism: Its Rites and Activities, $3^{\mathrm{e}}$ édition ( $1^{\text {re }}$ édition 1939), Bangkok, Suriyabun Publishers, $331 \mathrm{p}$.

WYATT, Robert K.

1976. - "Chronicle Traditions in Thai Historiography", Southeast Asian History and Historiography: Essays presented to D. G. E. Hall, Cowan, C. D. and Wolters, O.W. (ed.), Ithaca, NY, Cornell University Press, p. 107-122.

1984 - Thailand a Short History, London, Yale University Press, and Bangkok, Thai Watana Panich.

ZAGO, Marcel O.M.I.

1972 - Rites et cérémonies en milieu bouddhiste lao, Documenta Missionalia 6, Rome, Università Gregoriana Editrice; 408 p. 